\title{
A 60-Gb/s PAM4 Wireline Receiver With 2-Tap Direct Decision Feedback Equalization Employing Track-and-Regenerate Slicers in 28-nm CMOS
}

\author{
Kuan-Chang Chen ${ }^{\circledR}$, Member, IEEE, William Wei-Ting Kuo, Graduate Student Member, IEEE, \\ and Azita Emami ${ }^{\circledR}$, Senior Member, IEEE
}

\begin{abstract}
This article describes a 4-level pulse amplitude modulation (PAM4) receiver incorporating continuous time linear equalizers (CTLEs) and a 2-tap direct decision feedback equalizer (DFE) for applications in wireline communication. A CMOS track-and-regenerate slicer is proposed and employed in the PAM4 receiver. The proposed slicer is designed for the purposes of improving the clock-to- $Q$ delay as well as the output signal swing. A direct DFE in a PAM4 receiver is made possible with the proposed slicer by having rail-to-rail digital feedback signals available with reduced delay, and accordingly relaxing the settling time constraint of the summer. With the 2-tap direct DFE enabled by the proposed slicer, loop-unrolling and inductor-based bandwidth enhancement techniques, which can be area/power intensive, are not necessary at high data rates. The PAM4 receiver fabricated in 28-nm CMOS technology achieves bit-error-rate (BER) better than 1E-12, and energy efficiency of $1.1 \mathrm{pJ} / \mathrm{b}$ at $60 \mathrm{~Gb} / \mathrm{s}$, measured over a channel with 8.2-dB loss at Nyquist.
\end{abstract}

Index Terms-Comparator, decision-feedback equalizer (DFE), equalization, 4-level pulse amplitude modulation (PAM4), receiver, slicer, wireline.

\section{INTRODUCTION}

$\mathbf{F}$ OUR-LEVEL pulse amplitude modulation (PAM4) signaling has become an attractive option for high-speed data communication links where the channels suffer from severe bandwidth limitation, by virtue of its halved Nyquist frequency in comparison with that of non-return-to-zero (NRZ) modulation. In other words, the PAM4 signaling improves the spectral efficiency over that of NRZ, by encoding two bits of information, often referred to as the most significant bit (MSB) and the least significant bit (LSB), into one symbol. The consequent advantages of using PAM4 as a substitution for NRZ include the following: the bandwidth requirements for the channel and the front-end circuits are both reduced, and the circuits for clock generation and distribution can operate at the halved frequency. These advantages can potentially lead to higher data rates and/or lower power consumptions. However, there are new challenges stemming from the nature of multilevel signaling when designing PAM4 transceivers.

Manuscript received June 2, 2020; revised August 8, 2020; accepted September 6, 2020. This article was approved by Guest Editor Qun Jane Gu. (Corresponding author: Kuan-Chang Chen.)

The authors are with the California Institute of Technology, Pasadena, CA 91125 USA (e-mail: kcxchen@caltech.edu).

Color versions of one or more of the figures in this article are available online at http://ieeexplore.ieee.org.

Digital Object Identifier 10.1109/JSSC.2020.3025285
Specifically, with a fixed transmitter swing that is divided into the multiple levels, the receiver needs to resolve the transmitted bits from signals that have lower strength. The foregoing infers to two important design challenges, which this work focuses to address. One is the more demanding sensitivity of the decision circuitry, as will be elaborated in later paragraphs. The other is the necessity of canceling the inter-symbol interference (ISI), since the ISI resulting from strong symbols, for example, $(\mathrm{MSB}, \mathrm{LSB})=(+1,+1)$, can intrude detrimental interference on the nearby weak symbols, for example, $(\mathrm{MSB}, \mathrm{LSB})=(-1,+1)$, and cause undesirable data eye closure as a result. The same proportion of ISI level can be, on the contrary, tolerable in the cases of NRZ modulation in that the bipolar symbols, or bits, have nominally identical magnitude of signal swings.

Depending on the architecture of the receiver, analog-based equalization and/or analog-to-digital converter (ADC)-based equalization can be employed. In both scenarios, the incorporation of a decision feedback equalizer (DFE) is often an appealing option, as a DFE can succeed in compensating post-cursor ISI without amplifying crosstalk and noise. Recent examples include an ADC-based PAM4 receiver [1], designed in 16-nm FinFET CMOS, utilizing analog CTLE together with 24-tap feedforward equalizer (FFE) and 1-tap DFE implemented in digital domain, and the transceiver achieves biterror-rate (BER) less than $1 \mathrm{E}-8$ at $56 \mathrm{~Gb} / \mathrm{s}$ over a channel with 31-dB loss at $14 \mathrm{GHz}$ (Nyquist frequency). With the feasibility of integrating hybrid analog and digital equalization including long-tap FFE, ADC-based receiver architectures have been designed for longer reach or channels with loss greater than $30 \mathrm{~dB}$ at Nyquist [1]-[4]. On the other hand, an analog-based 40-56-Gb/s PAM4 receiver in 16-nm FinFET CMOS [5], targeting chip-to-module and board-to-board cable interconnects, mitigates the channel loss of $10 \mathrm{~dB}$ at $14 \mathrm{GHz}$ and reflections, by incorporating CTLE and direct 10-tap DFE in analog domain. Compared to [1] that equalizes $>30-\mathrm{dB}$ loss at Nyquist with ADC-based architecture, this analogbased receiver [5] designed for 10-dB loss at Nyquist achieves BER of less than $1 \mathrm{E}-12$ at $56 \mathrm{~Gb} / \mathrm{s}$, but consumes $40 \%$ less power [5]. These previous designs suggest that for short reach applications where channel losses can be less than $10 \mathrm{~dB}$ at Nyquist, an ADC-based receiver may not be the optimal solution in consideration of both the hardware and power that need to be invested. 


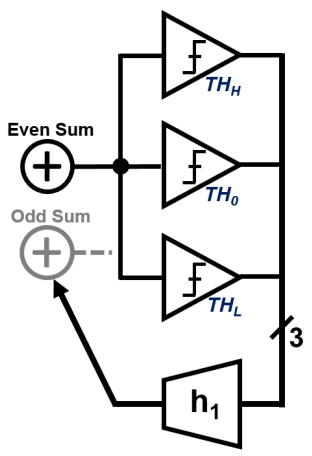

Direct 1-Tap PAM4-DFE

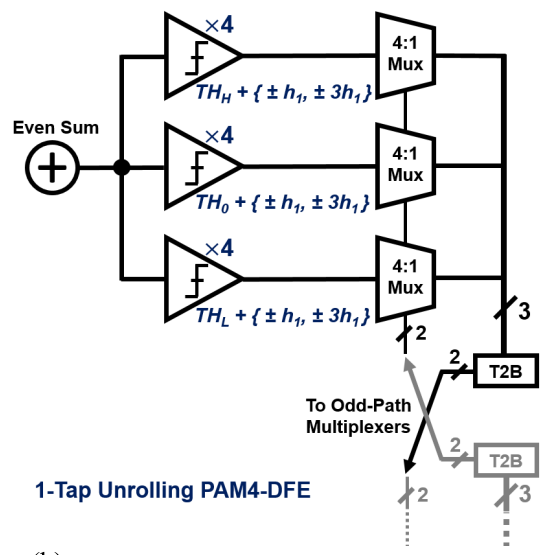

(b) (a)

Fig. 1. Hardware implementation of PAM4-DFE in half-rate designs. Only the even data-path is shown for clarity, where $\mathrm{TH}_{\mathrm{H}}, \mathrm{TH}_{0}, \mathrm{TH}_{\mathrm{L}}$ are the three distinct threshold levels, and $h_{1}$ corresponds to the first post-cursor ISI. (a) Direct 1-tap PAM4-DFE. (b) Loop-unrolling 1-tap PAM4-DFE.

Despite the usefulness of including a DFE as part of a PAM4 receiver in the analog fashion, as demonstrated in [5]-[7], improving the energy efficiency of an analogbased PAM4-DFE at high data rates remains challenging. First, compared with NRZ receivers, the reduced eye-height in PAM4 receivers (by a factor of $\sim 3$ in the absence of nonlinearity and with fixed transmitter swing) sets a more stringent limit for the sensitivity of the slicer used for resolving the symbols and making decisions. Furthermore, the sensitivity requirement generally becomes more difficult to meet, given tighter timing constraints, such as at higher data rates and/or with lower decision latency requirements in feedback loops. Second, at least three slicers are required with respect to the three distinct thresholds, and therefore, the power consumed by the slicers and the loading presented by the slicers are of much greater concern in designing PAM4 receivers. Moreover, as can be seen in Fig. 1, which compares the implementation of direct 1-tap PAM4-DFE with that of 1-tap loop-unrolling PAM4-DFE, the loop-unrolling technique demands significantly more hardware. Even if only one tap is unrolled, it needs 12 slicers, three multiplexers, and one thermometer-to-binary (T2B) decoder for each deserialized branch (e.g., 24 slicers, six multiplexers, and two T2B decoders in total for a half-rate design). Since the number of slicers increases exponentially with the number of taps unrolled, the loop-unrolling technique is prohibitively costly in hardware and power consumption for a high data rate PAM4 receiver, suggesting that the speed or the delay performance of the slicers is critical. As illustrated in Fig. 2, a stringent timing constraint that requires all the operations to be finished within $1 \mathrm{UI}$ is set, when attempting to directly close the decision feedback loop for the first tap. Although the signal propagation and settling happen concurrently in reality, it is informative and useful to conceptually distinguish them into the setup time of the slicer, clock-to$\mathrm{Q}$ delay of the slicer, the propagation delay of the DFE tap, and the settling time of the summer. Details and interpretations of these timing constraints are presented in Section IV. In particular, since the clock-to-Q delay of the slicer takes up a considerable portion in the 1-UI constraint, as will be

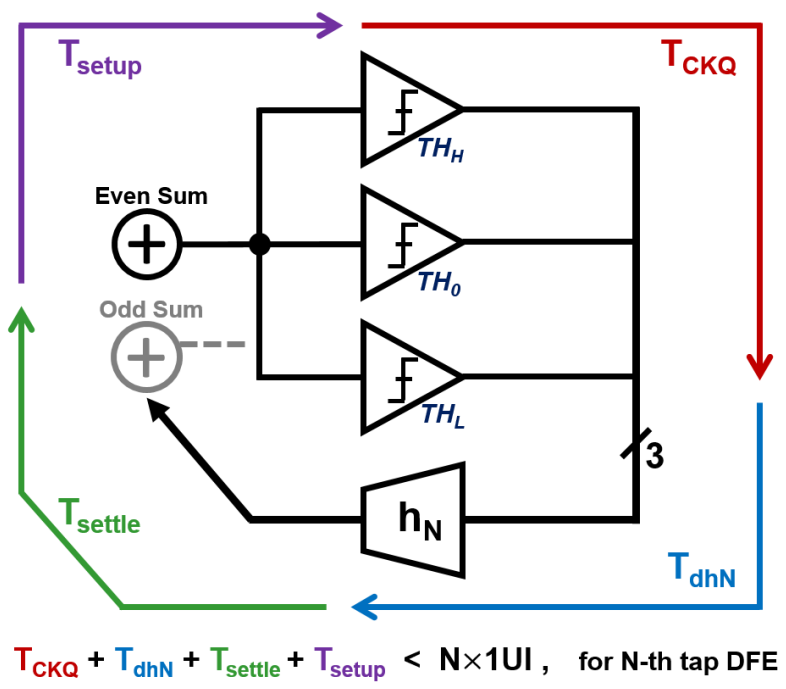

Fig. 2. Timing constraint for a direct DFE design for $N$ th post-cursor ISI compensation.

shown in Section III, the improvement in slicer delay helps to close the loop at higher data rates or to relax the summer design such that no excess power-bandwidth tradeoffs or areaconsuming inductors are required for reducing the summer settling time. Therefore, this work aims to demonstrate the idea of implementing an energy-efficient PAM4 receiver with direct DFE loops by improving the slicer performance.

This article, expound upon [22], is organized as follows. Section II presents the overall PAM4 receiver architecture, where each subsection describes the circuits that serve as key building blocks in the analog front-end (AFE) and in the clock path. Section III reviews the operations and features of prevalent slicer topologies, and describes the proposed slicer in detail. Section IV elaborates the timing constraint for completing the DFE loops. Experimental results of this PAM4 receiver are shown in Section V, and finally, Section VI summarizes this work with performance comparisons and conclusions.

\section{RECEIVER ARCHIteCture}

\section{A. Overall Architecture}

The overall architecture of the PAM4 receiver is shown in Fig. 3. The AFE is composed of two stages of continuous time linear equalizer (CTLE) and two half-rate summers. The outputs of each summer are connected to four proposed CMOS track-and-regenerate slicers, among which one is responsible for the eye monitor (EM), and the other three slicers are dedicated to recovering the analog summer outputs to the corresponding 3-bit thermometer-coded digital levels. With the proposed slicers, direct 2-tap DFE is implemented. The 3-bit thermometer-coded outputs are first directly fed back to the summer in the other data path for the first tap of DFE, and then with 1-UI delay, fed back to the summer in the same data path for the second tap of DFE. The digital-level slicer outputs are further demultiplexed (1-to-32) for external and on-chip EM and BER counters (BERCs) to evaluate the eyeopening and BER performance, respectively. The clock path 


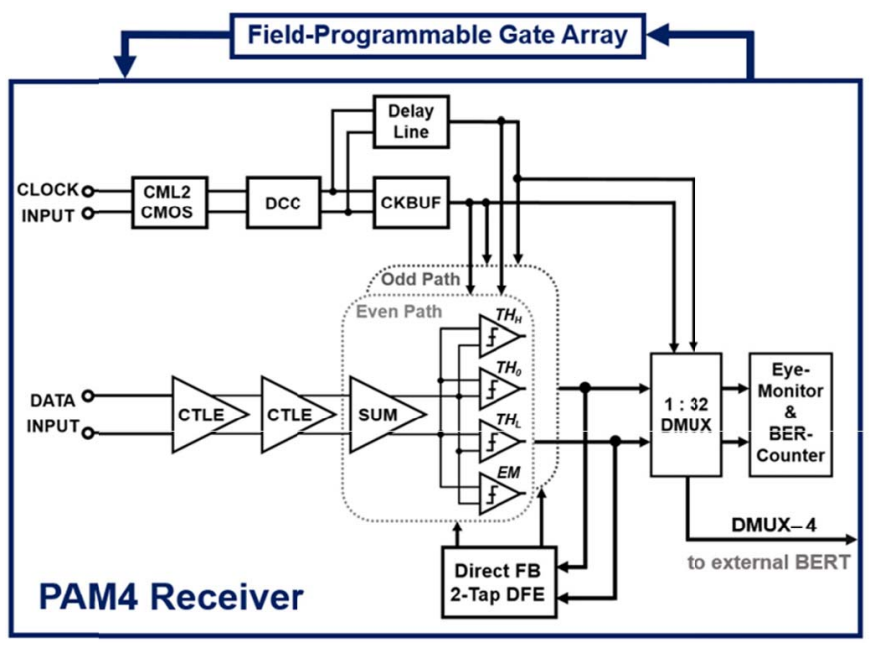

Fig. 3. Overall architecture of the PAM4 receiver.

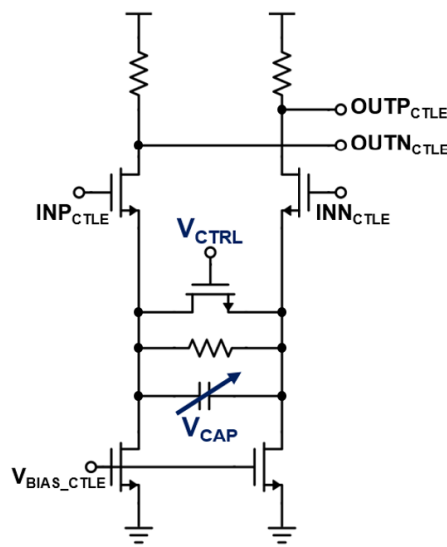

(a)

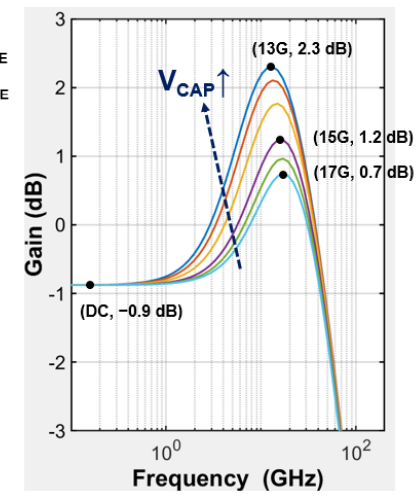

(b)

Fig. 4. (a) Schematic of the source-degenerated CTLE. (b) Simulated frequency response of the CTLE (single stage) with different settings of $V_{\mathrm{CAP}}$.

takes in an external pair of half-rate differential clock signals and amplifies them to rail-to-rail levels with on-chip duty cycle correction (DCC). Clock buffers (CKBUFs) and a digitally adjustable delay line (DL) are included on the chip, serving as the interfaces with the clocked slicers to provide rail-to-rail clock signals for data recovery as well as the required clock phases for eye monitoring.

The following sections describe the details associated with the design of CTLE in Section II-B, half rate summers in Section II-C, linearity characterizations in Section II-D, current mode logic (CML)-to-CMOS clock converter in Section II-E, and DCC circuits in Section II-F. The details of the proposed slicer are presented in Section III.

\section{B. CTLE}

CTLE is included in the receiver to mitigate both pre-cursor ISI and post-cursor ISI, as the coverage of the direct 2-tap DFE design is limited to the first and second post-cursor ISI. Fig. 4(a) shows the schematic of the CTLE, which adopts the conventional topology of $R C$ source-degenerated differential amplifier with digital controllability. The high-frequency peaking can be enabled or disabled by setting $V_{\text {CTRL }}$ to be

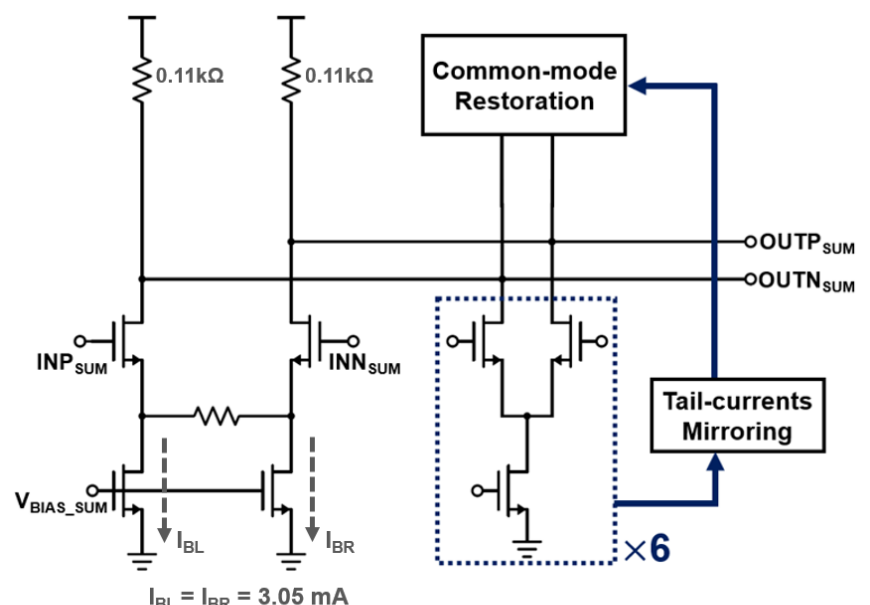

\begin{tabular}{|c|c|c|}
\hline Small-Signal Gain & -3dB Bandwidth & $\mathbf{9 5 \%}$ Settling Time \\
\hline $0.9 \mathrm{~V} / \mathrm{V}$ & $34.45 \mathrm{GHz}$ & $13.68 \mathrm{ps}$ \\
\hline
\end{tabular}

Fig. 5. Architecture and performance of the summer for 2-tap DFE.

logic low or logic high, respectively. As shown in Fig. 4(b), the peaking frequency is digitally adjustable by varying the voltage level of $V_{\mathrm{CAP}}$. Since the source-degenerated resistance remains unchanged, the dc gain of the CTLE is approximately $0.9(\mathrm{~V} / \mathrm{V})$, independent of the setting of $V_{\mathrm{CAP}}$. Without the inclusion of inductors, the frequency boost at $15 \mathrm{GHz}$ is simulated to be $2.1 \mathrm{~dB}$ for a single CTLE stage. The voltage level of $V_{\text {CAP }}$ is set by an 8-bit on-chip voltage digital-toanalog converter (DAC), and the implementation of which follows the conventional resistor ladder R-2R architecture as presented in [1]. The voltage DAC therefore provides a dc voltage with 8-bit resolution between the ground $(0 \mathrm{~V})$ and a reference voltage, $V_{\mathrm{HIGH}}$, where the value of $V_{\mathrm{HIGH}}$ can be changed via a pad connected to an external voltage source. In this prototype, an on-chip voltage DAC bank consisting of duplications of the aforementioned 8-bit voltage DAC is responsible for generating the digitally adjustable voltage levels. For further reduction in the area overhead, the resolution of each voltage DAC can be individually optimized with respect to the associated circuit blocks.

\section{Summer}

The summers used in the PAM4 receiver fall in the category of resistively loaded CML summer, and the architecture incorporating 2-tap DFE summation is shown in Fig. 5. Resistive source-degeneration is employed for linearity improvement. Depending on the previous two symbols resolved by the three data slicers; that is, the corresponding six thermometer-coded digital signals in differential fashion, the six tail currents are respectively steered to one of the two load resistors to perform DFE summation. To maintain the common-mode voltage level at the summer outputs irrespective of the DFE setting, all these tail currents are summed and mirrored to a common-mode restoration block which injects the currents evenly from the supply into the summing nodes (OUTP SUM $_{\text {SM }}$ aUTN $\left.\mathrm{OUU}_{\text {SUM }}\right)$. The common-mode restoration allows the threshold setting and delay performance of the slicers to be independent of the DFE setting. 


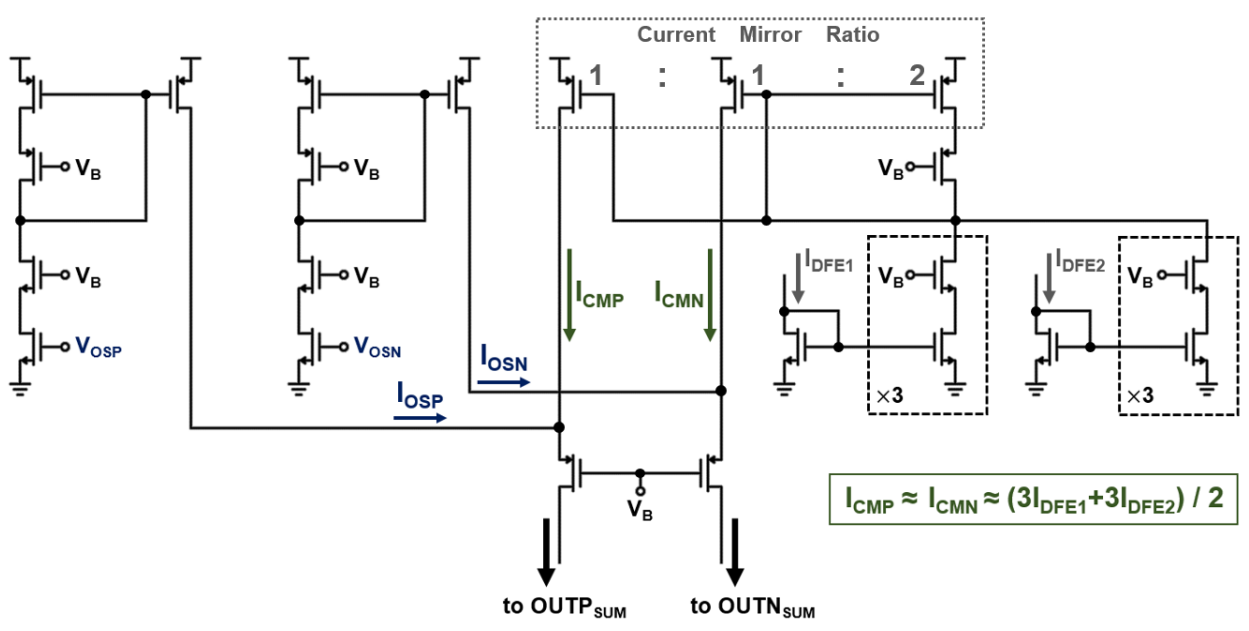

(a)

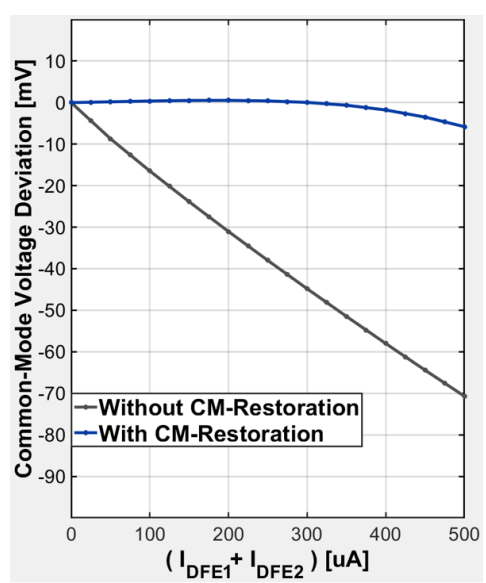

(b)

Fig. 6. (a) Schematic of the common-mode restoration circuits. (b) Simulated performance of the common-mode restoration circuits, showing the deviation from the target common mode with and without the common-mode restoration circuits.

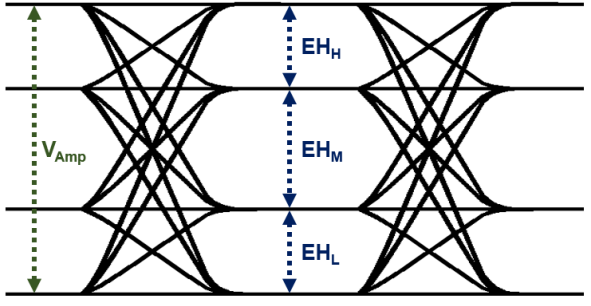

Eye Linearity $(\mathrm{EL})=\frac{\min \left(\mathrm{EH}_{\mathrm{H}}, \mathrm{EH}_{\mathrm{M}}, \mathrm{EH}_{\mathrm{L}}\right)}{\max \left(\mathrm{EH}_{\mathrm{H}}, \mathrm{EH}_{\mathrm{M}}, \mathrm{EH}_{\mathrm{L}}\right)}$

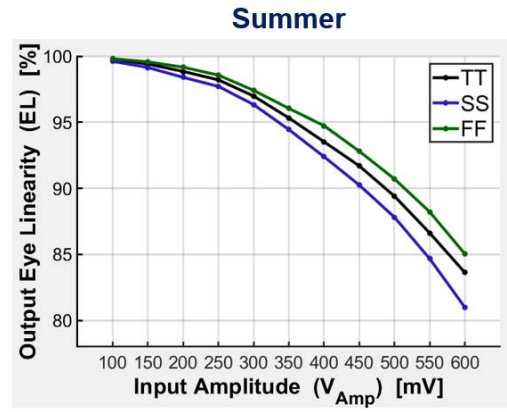

(b)

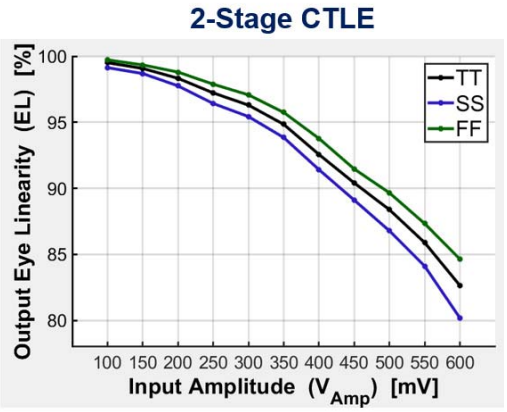

(c)

Fig. 7. (a) Nomenclature for PAM4 eye diagrams and the definition for PAM4 EL. (b) Simulated linearity performance of the summer. (c) Simulated linearity performance of the CTLE.

The schematic of the common-mode restoration circuits is shown in Fig. 6(a). It is similar to that in a prior art [5], while an additional function is included in this work for offset compensation. The common-mode restoration currents, $I_{\mathrm{CMP}}$ and $I_{\mathrm{CMN}}$, are nominally half of the sum of all DFE currents that is, $\left(3 I_{\mathrm{DFE} 1}+3 I_{\mathrm{DFE} 2}\right) / 2$. The offset cancellation currents, $I_{\mathrm{OSP}}$ and $I_{\mathrm{OSN}}$, are individually adjustable to compensate the accumulated dc offset of the CTLE stages and the summer. In this prototype, a closed offset-cancellation loop is not implemented, and the values of $I_{\mathrm{OSP}}$ and $I_{\mathrm{OSN}}$ are adjusted with on-chip voltage DACs. Due to the finite output resistance of the current sources and current mirrors, larger errors can be introduced when the currents to be copied become larger. As shown in Fig. 6(b), simulations have been carried out to study the deviations from the target common-mode voltage level, with distinct settings of DFE currents. It can be seen that without the common-mode restoration circuits, the output common-mode level of the summer drops roughly linearly with the increase of DFE currents; the voltage drop of output common-mode level is approximately $70 \mathrm{mV}$ when $\left(I_{\mathrm{DFE} 1}+I_{\mathrm{DFE} 2}\right)=500 \mu \mathrm{A}$. By contrast, when the common-mode restoration circuits are connected, the voltage drop of output common-mode level is less than $6 \mathrm{mV}$ when $\left(I_{\mathrm{DFE} 1}+I_{\mathrm{DFE} 2}\right)=500 \mu \mathrm{A}$, and a relatively constant output common-mode level is sustained across the range shown in Fig. 6(b).

\section{Linearity Characterizations}

Since the receiver front-end linearity performance is crucial for multilevel signaling, the linearity of the summer and that of the CTLE are respectively examined via the evaluations on the output eye linearity (EL) versus the input amplitude. Fig. 7(a) shows the nomenclature for PAM4 eye diagrams along with the definition used for PAM4 EL, where $V_{\text {Amp }}$ denotes the peak-to-peak input amplitude; $\mathrm{EH}_{\mathrm{H}}, \mathrm{EH}_{\mathrm{M}}$, and $\mathrm{EH}_{\mathrm{L}}$ measure the eye heights of the upper eye, the middle eye, and the lower eye, respectively. Clean PAM4 signals without level mismatch (i.e., $\mathrm{EL}=1$ ) are applied to the input of the summer, and the output EL of the summer is recorded for each given input amplitude. Similarly, to test the CTLE linearity, PAM4 signals of different amplitudes with EL $=1$ are generated, whereas these signals go through a channel with $4-\mathrm{dB}$ loss at $15 \mathrm{GHz}$ before being applied to the input of the CTLE. The two-stage CTLE is correspondingly configured to provide $\sim 4-\mathrm{dB}$ boost at $15 \mathrm{GHz}$, which is the target amount of peaking, as described in Section II-B. The simulated results at different process corners are shown in Fig. 7(b) for the summer, and Fig. 7(c) 


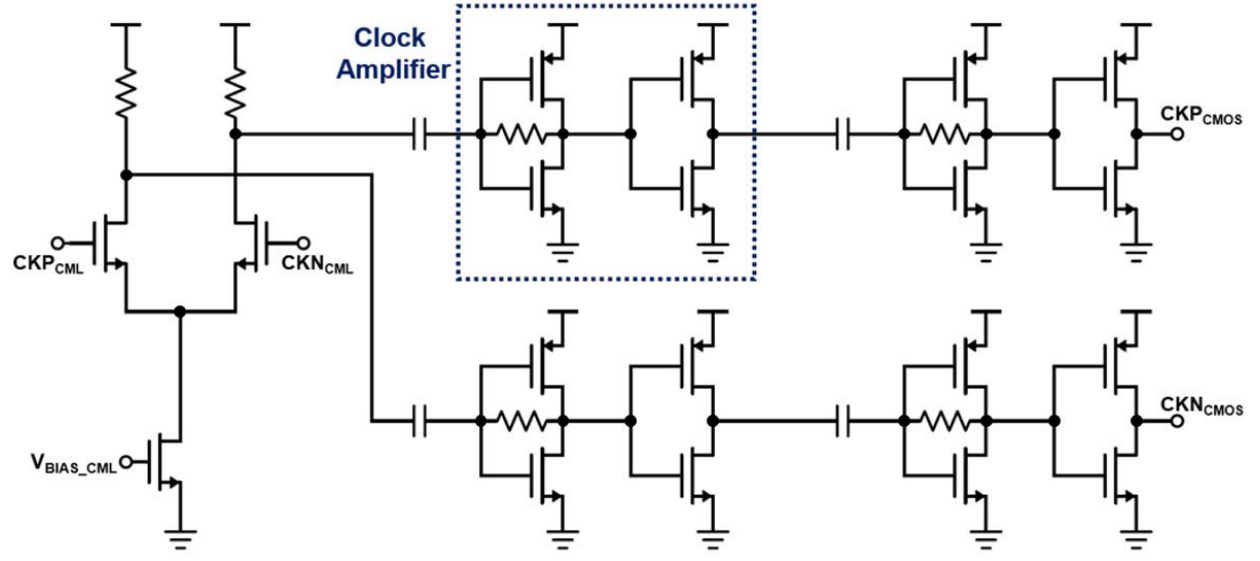

(a)

Fig. 8. (a) Schematic of the CML-to-CMOS clock converter. (b) Simulated
frequencies for the CML-to-CMOS clock converter.
for the CTLE. As variable gain amplifiers (VGAs) are not included in this prototype, the EL remains above $90 \%$, only when $V_{\text {Amp }}$ is not greater than $\sim 450 \mathrm{mV}$.

\section{E. CML-to-CMOS Clock Converter}

Fig. 8(a) shows the schematic of the CML-to-CMOS clock converter. It consists of a differential amplifier and two stages of ac-coupled inverter-based clock amplifier. The use of ac coupling capacitor and inverter with the input node connected to the output node via a resistor ensures that the dc level of the clock signals is biased to around half of the supply voltage. The CML-to-CMOS clock converter is able to amplify incoming sinusoidal clock signals to rail-to-rail (i.e., CMOS levels) at various clock frequencies, provided that the amplitude of the input sinusoidal signals is sufficiently large. Fig. 8(b) summarizes the minimum required peak-to-peak amplitudes at different frequencies such that the swings of the clock signals at the converter output are larger than $50-850 \mathrm{mV}$. In particular, for $15-\mathrm{GHz}$ clock signals, $24-\mathrm{mV}_{\mathrm{pp}}$ input amplitude is needed for output swing larger than $50-850 \mathrm{mV}$, and $40-\mathrm{mV}_{\mathrm{pp}}$ input amplitude further increases the output swing from approximately ground $(0 \mathrm{~V})$ to the supply voltage (900 mV). By providing larger input amplitudes, this CMLto-CMOS clock converter can work at higher frequencies.

\section{F. DCC Circuits}

Duty-cycle distortion effectively induces unequal time frames for the operations (e.g., sampling, or data recovery) in different data paths, and therefore duty-cycle distortion can be highly undesirable for high data-rate designs where the performance such as BER is sensitive to the unwanted reduction or imbalance of the timing allocation. In light of the negative effects of the duty-cycle distortion, DCC circuits are designed and implemented on the chip. Fig. 9(a) presents the schematic of the DCC circuits. The duty-cycle is adjusted by varying the amounts of the currents, $I_{\mathrm{UP}}$ and $I_{\mathrm{DN}}$, which are digitally programmable by 10 bits, $b<9: 0>$. In addition, to be capable of accommodating both large duty-cycle distortion and finetuning, the value of $V_{\mathrm{BIAS}}$, which sets the current level of the

\section{A. Overview}

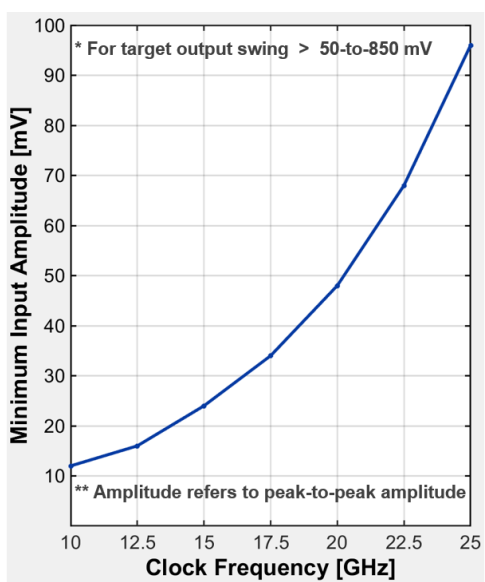

(b)

current sources, is designed to be also digitally adjustable with an on-chip DAC. Simulation results of the DCC at $15 \mathrm{GHz}$ are shown in Fig. 9(b). With the simultaneous programmability of the values of $I_{\mathrm{UP}}, I_{\mathrm{DN}}$, and $V_{\mathrm{BIAS}}$, the DCC is able to correct the input clock signal with duty-cycle of $25 \%-75 \%$ such that the duty-cycle of the output clock signal is very close to $50 \%$ with errors not greater than $0.1 \%$. Provided that the duty-cycle of the input clock source to the receiver chip is $50 \%$, Monte Carlo simulations show that the resultant duty-cycle at the outputs of the on-chip clock path varies from $48.46 \%$ to $52.48 \%$. Accordingly, the presented DCC well covers the range due to process variations, and also has the competence to accommodate an input clock source whose duty-cycle deviates from $50 \%$. In this work, an on-chip adaptive closed loop for setting the DCC is not implemented, but the setting is swept with the aim of optimizing the measured BER instead.

\section{SLICER DESIGN}

Voltage comparators, also known as slicers, or sense amplifiers in some contexts, have served widely in mixed-signal circuits and systems, including ADCs, adaptive configuration loops, memory access circuitry, and data receivers. A variety of slicer topologies with their practical utility have been demonstrated. In [8], CML slicers appeared in the implementation of a 6-bit ADC, and later the CML slicer topology has been frequently employed in data receivers [6], [7], [9], [10]. A CMOS latch-type comparator, famous as the StrongArm and originally studied in memory circuits [11], becomes popular due to its often negligible static power consumption and the competence to generate rail-to-rail output swings. The StrongArm has found broad applications in both low-power architectures and high-speed receivers, and its mechanism appears to incite inventions or variants of CMOS latch-type slicers. A 2-stage topology called double-tail latch-type voltage sense amplifier is presented in [12], which enhances the capability of operating at lower supply voltages and input common-mode voltage levels, by having less stacking transistors and separate tail currents for the input stage and the latch stage. The slicers 


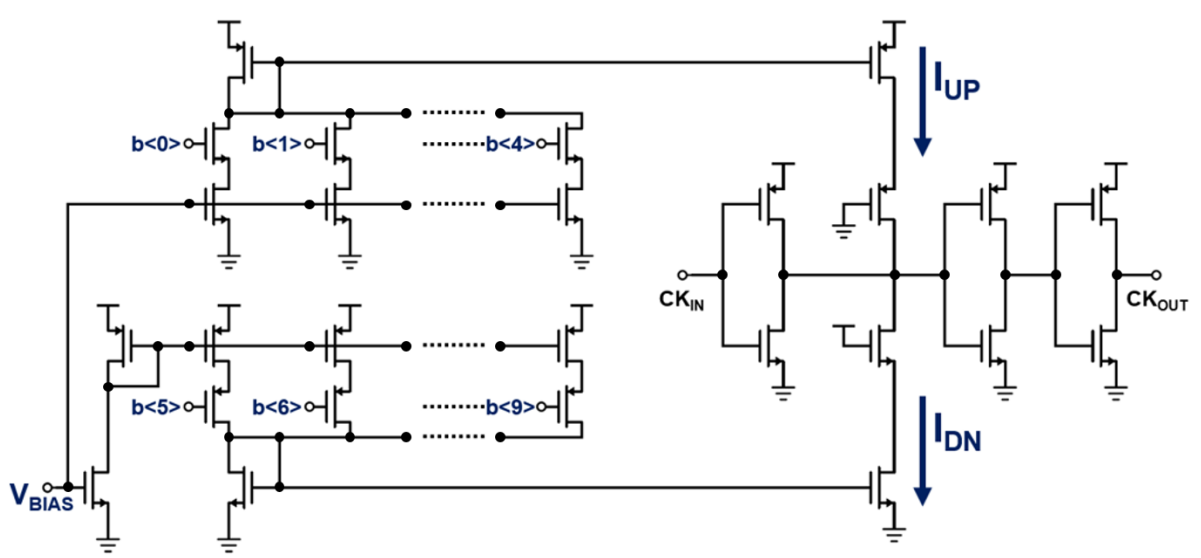

(a)

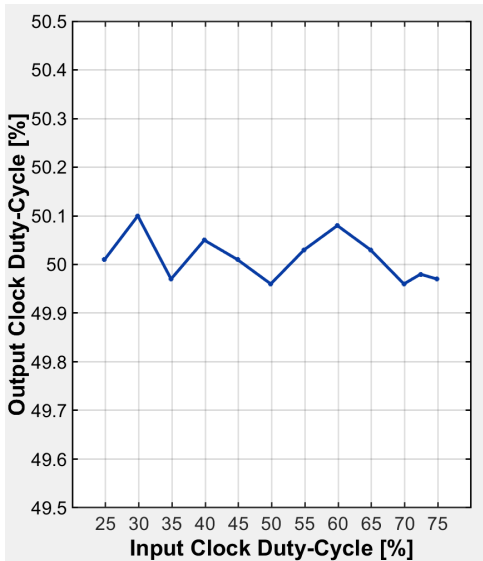

(b)

Fig. 9. (a) Schematic of the DCC circuits. (b) Simulated performance of DCC with 15-GHz clock signals.

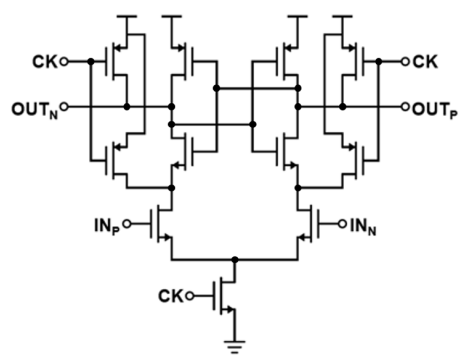

(a)

(b)

Fig. 10. Prevalent slicer topologies. (a) StrongArm slicer. (b) CML slicer.

used in [13] and [14] are both essentially variants of the double-tail latch-type slicer [12], with an augmented function to incorporate 1-tap DFE summation. Another 2-stage slicer is reported in [15], where it is mentioned that increasing the common mode for the same clock-to-Q delay is enabled. Compared with the StrongArm, the aforementioned latch-type slicers [12]-[15] attempt to conform the delay performance among an extended range of supply voltage or input commonmode levels, without much emphasis on considerably reducing the achievable delay.

As illustrated in Fig. 2, the clock-to-Q delay performance of the slicers plays a critical role in closing the DFE loops. The next subsection describes the features of the particularly prevalent two slicer circuits; that is, the StrongArm and the CML slicer, and discusses the potential improvements.

\section{B. Prevalent Slicer Topologies}

The schematic of the StrongArm is shown in Fig. 10(a). It is designed in a dynamic CMOS latch fashion and its typical operation is illustrated in Fig. 11. When the clock (CK) is logic low, the outputs are both being charged to the supply value such that the differential output is reset to approximately zero. When the clock becomes logic high, the StrongArm samples the differential input and then the differential output is regenerated toward rail-to-rail with the help of the positive feedback offered by the cross-coupled pairs. A few observations can be made after closely examining the simulated waveforms. First,

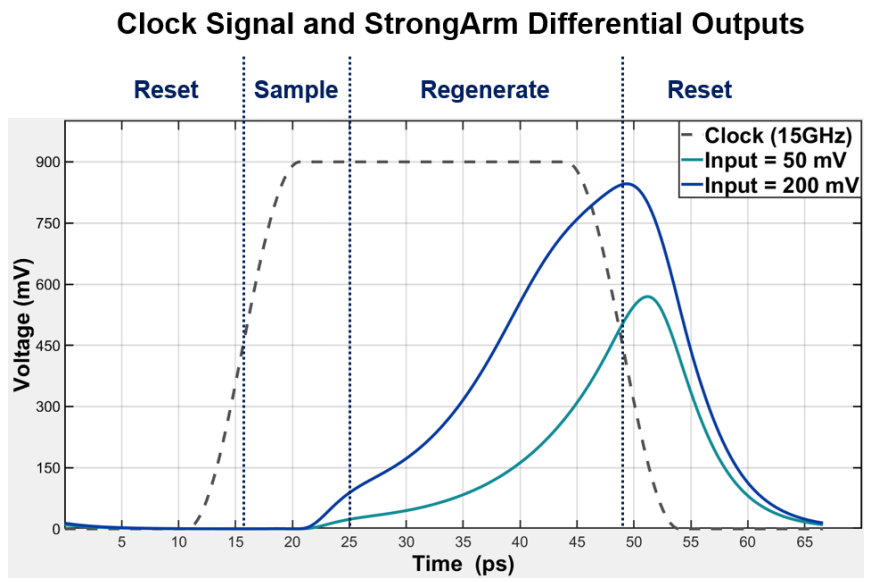

Fig. 11. Simulated waveforms showing the typical operations including the reset, sample, and regenerate phases of the StrongArm slicer.

attributing to the reset mechanism, there is always certain time that needs to be spent for the differential output signal to grow from approximately zero. Second, since the time allocated for regeneration is limited by the data rate, the regeneration started with a higher level is very beneficial in that at the end of the regeneration phase, the differential output swing can be considerably larger and the delay to achieve digital level is also significantly less. In other words, for high data-rate operations, the time required for the output signals of the StrongArm to grow from approximately zero to the level that can be identified as digital outputs may not be sufficient. The above observations motivate the idea to design a slicer which instead of resetting, tracks the polarity of the differential input signal such that the regeneration can proceed with a higher signal level. Another prevalent slicer topology shown in Fig. 10(b), known as the CML slicer, leverages the idea of tracking the inputs. However, as the output swing magnitude of the CML slicer cannot exceed the product of the tail current and the load resistance, $\left(I_{\text {TAIL }} \times R_{\mathrm{L}}\right)$, a number of drawbacks are associated with the CML slicer when implementing a PAM4DFE with it. For one thing, since the output swing is not rail-to-rail, the CML slicer may not be directly compatible to the relatively energy-efficient CMOS gates for delaying 
or buffering the resolved data, and a potential solution by inserting CML-to-CMOS amplifiers would increase the total delay. For another thing, the smaller output swing offers less strength to steer the DFE currents, and therefore, the sizes of the differential pairs of the DFE current branches cannot be minimized, which equivalently adds restrictions in minimizing the load capacitance at the summer outputs. Furthermore, referring to Fig. 10(b), because M1, M2 are directly connected to M3 and M4, when the CML slicer is designed for larger output swing with large tail current, it leads to relatively high power consumption, and at the same time presents large input and output capacitances.

\section{CMOS Track-and-Regenerate Slicer}

In view of the above, a CMOS track-and-regenerate slicer is proposed and designed, aiming to improve the clock-to-Q delay as well as the output swing. When the DFE is implemented with the proposed slicer, digital-level outputs are directly available and the settling time specification of the summer is relaxed in consequence of the reduced slicer delay, enabling an energy-efficient DFE design that operates at high data rates. The overall circuit schematic of the proposed CMOS track-and-regenerate slicer is shown in Fig. 12(a). The proposed slicer tracks the differential input instead of being reset, and it regenerates the differential output to rail-to-rail levels. Designed in CMOS dynamic latch fashion, the proposed slicer is suitable for technology scaling and can be viewed as having three-stage configuration. The first stage, consisting of M1-M10, works as a dynamic differential amplifier. M11-M14 form the second stage, which serves as a buffer to provide some isolation between the first and the third stage. The third stage, M15-M22, is essentially dynamically controlled cross-coupled pairs that are responsible for regenerating the signal with positive feedback. Fig. 12(b) and (c), respectively, illustrates the operation of the proposed slicer during the two complimentary clock phases. When CK is logic low and CKB is logic high, as in Fig. 12(b), M1-M8 and M11-M14 perform the tracking function with M9, M10, M17, and M18 turned off, and they overwrite the latch outputs $\left(\mathrm{OUT}_{\mathrm{P}}\right.$ and $\left.\mathrm{OUT}_{\mathrm{N}}\right) . \mathrm{M} 15$ and M16 are kept always on and conduct relatively weak currents to avoid the cross-coupled pairs (M19-M22) recovering from being completely off while allowing the outputs to be easily overwritten. In the other half of clock cycle, that is, when CK is logic high and CKB is logic low, as in Fig. 12(c), the tracking function is stopped with the outputs of the first stage being cleared and the second stage disabled. The outputs of the first stage are cleared by M9 and M10 which discharge the output node voltages toward zero, and hence eventually turn off M11 and M12. With M11 and M12 turned off by M9 and M10, respectively, M13 and M14 turned off with the rise of $\mathrm{CK}$, the second stage is quickly disabled, isolating the continuously changing inputs from the latch outputs which shall be regenerated toward rail-to-rail levels with respect to the polarity that has been tracked. At the same time, the cross-coupled pairs conduct significantly more currents by turning on M17 and M18, empowering strong positive

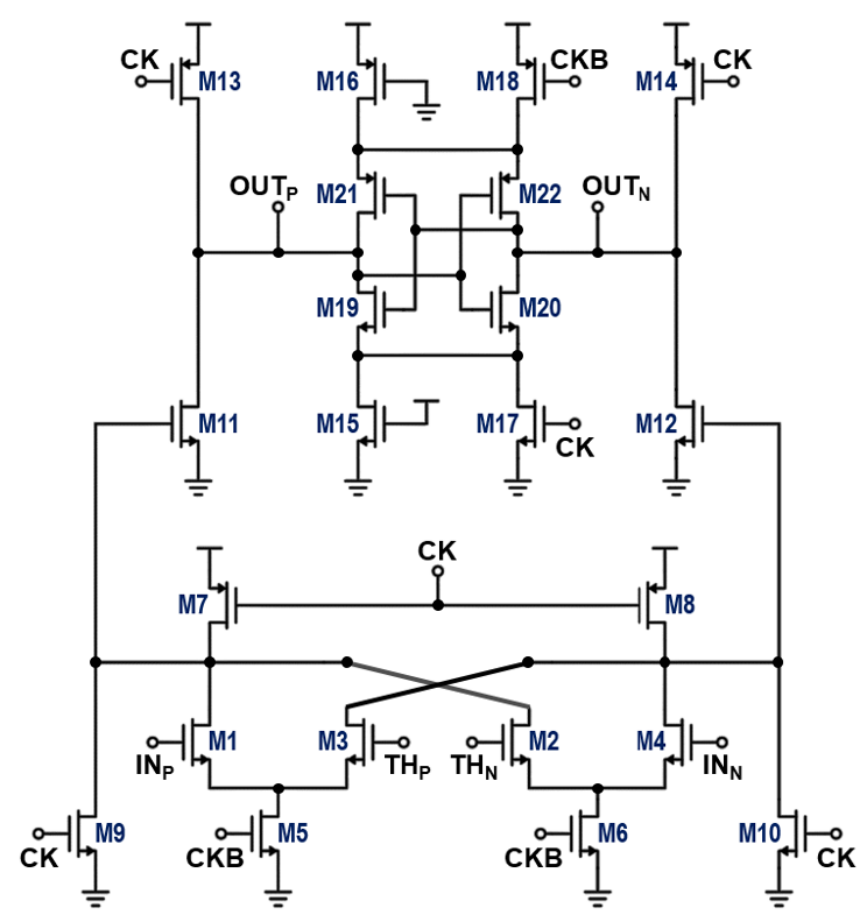

(a)

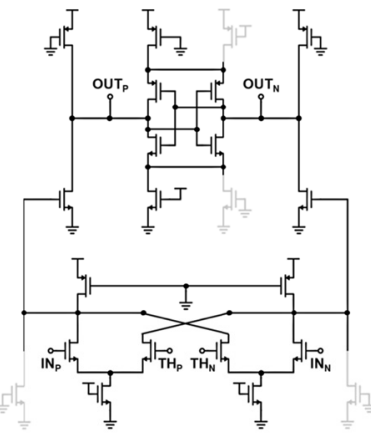

(b)

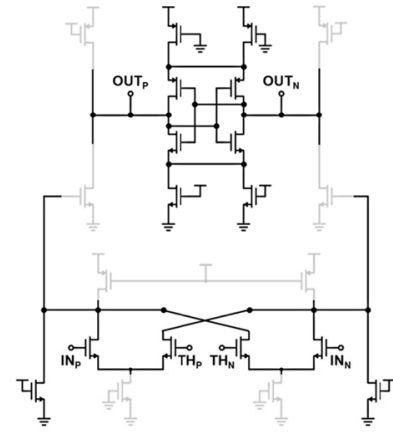

Regenerate Mode $(C K=1, C K B=0)$

(c)
Fig. 12. Proposed CMOS track-and-regenerate slicer. (a) Overall circuit schematic. (b) Proposed slicer in track mode. (c) Propose slicer in regenerate mode.

feedback for the regeneration. It is noteworthy that clearing the outputs of the first stage with M9 and M10 during the regenerate-mode also helps with tracking the inputs for the next tracking phase, thanks to that the first stage itself does not memorize the results from the previous tracking phase. In this work, the threshold level of the slicer is determined by the gate voltages $\mathrm{TH}_{\mathrm{P}}$ and $\mathrm{TH}_{\mathrm{N}}$, and each slicer has its own individually adjustable threshold generator. The threshold levels are programmable from an external field-programmable gate array (FPGA) and set by on-chip voltage DACs that are described in Section II-B. The slicer offset can be compensated by setting $\mathrm{TH}_{\mathrm{P}}$ and $\mathrm{TH}_{\mathrm{N}}$ correspondingly for a given threshold.

\section{Simulation Results}

For the purpose of demonstrating the features of the proposed CMOS track-and-regenerate slicer and comparing the 

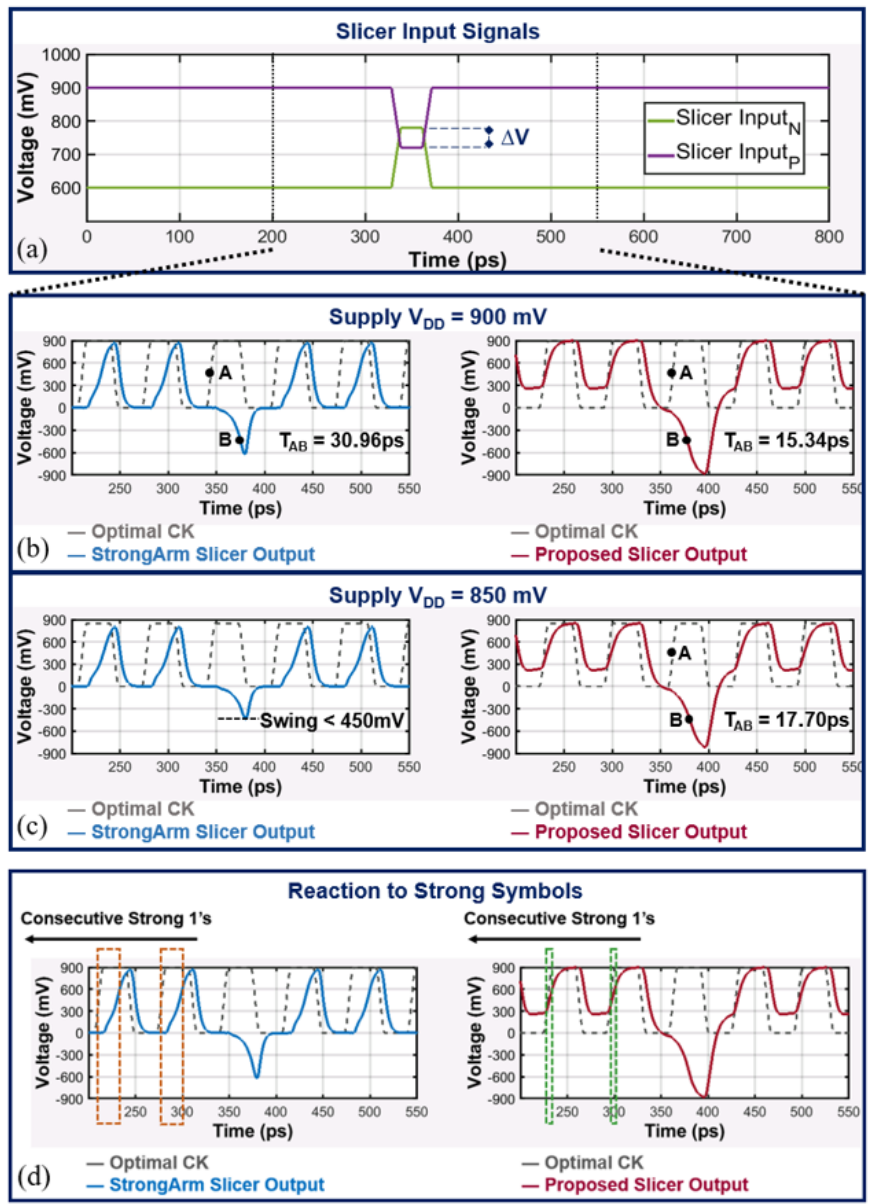

Fig. 13. Simulations and comparisons of the large-signal performance between the reset-and-regenerate StrongArm and the proposed CMOS trackand-regenerate slicer. (a) Input signals to the slicers. (b) Optimal clock signals and the resulting output waveforms of the slicers with $900-\mathrm{mV}$ supply. (c) Optimal clock signals and the resulting output waveforms of the slicers with $850-\mathrm{mV}$ supply. (d) Faster reaction to strong symbols with the proposed slicer.

proposed slicer with the StrongArm which is also compact in size and suitable for technology scaling, extensive simulations have been carried out and the results are presented as follows. Fig. 13 illustrates the large-signal behavior and clock-to-Q delay performance of the proposed CMOS "trackand-regenerate" slicer along with the "reset-and-regenerate" StrongArm slicer. The input signals to the slicers are shown in Fig. 13(a), representing a worst-case pattern when a weak negative symbol, that is, $(\mathrm{MSB}, \mathrm{LSB})=(-1,+1)$, is between a long sequence of strong positive symbols, i.e., (MSB, LSB $=(+1,+1)$. Using this input pattern, the worst-case delay performance for PAM4 signaling can be evaluated and the memory effect in the proposed slicer with non-resetting mechanism is examined. As shown in Fig. 13(b), in contrast to the conventional CML slicer, the proposed slicer offers railto-rail output swings and thus direct availability of digital-level outputs. Meanwhile, in comparison with the StrongArm slicer, instead of resetting the latch, the proposed slicer tracks the input signals like how the CML slicer does, helping to reduce the required regeneration time. As a result, the proposed slicer improves the clock-to-Q delay as well as the output swing over the StrongArm. With the sizes of the input transistors and the output cross-coupled pairs designed to be identical, the worst-case clock-to-Q delay (with respect to the switching points defined as $\pm 450 \mathrm{mV}$ ) is simulated to be $30.96 \mathrm{ps}$ for the StrongArm, whereas the delay reduces to $15.34 \mathrm{ps}$ for the proposed slicer. As already been shown in [21], when not operating with low supply voltages or low input common-mode levels, the delay performance of the doubletail slicer [12] is similar to that of the StrongArm. This is also observed when a double-tail is tested with the same data pattern shown in Fig. 13(a). The double-tail slicer is designed to have the same input stage and output cross-coupled pairs as the StrongArm, achieving $31.2 \mathrm{ps}$ with the input commonmode level set to $750 \mathrm{mV}$, and $29.58 \mathrm{ps}$ with the input common-mode level reduced to $600 \mathrm{mV}$, for the worst-case clock-to-Q delay. Fig. 13(c) furthermore shows the immunity to the change in the power supply. A voltage drop of $50 \mathrm{mV}$ from a $900-\mathrm{mV}$ supply hinders the StrongArm from resolving the weak negative symbol to digital level with its output swing less than $450 \mathrm{mV}$, while the output swing of the proposed slicer is still approximately rail-to-rail and the penalty on resolving the weak symbol is 2.36 ps of increase in delay. Fig. 13(d) emphasizes another desirable feature offered by the proposed slicer; namely, the fast reaction to strong symbols. Since the strong symbols tend to cause relatively strong ISI for the next symbols, it is beneficial to have fast reaction and thus fast decision on them. The fast reaction makes sure the DFE summation is completely settled so as to minimize the negative impact of the residual ISI caused by the strong symbols.

In addition to the improved clock-to-Q delay performance, the proposed slicer also holds superior output swing and input sensitivity to the StrongArm, as can be seen below. Fig. 14(a) shows the input pattern under tests, which is similar to Fig. 13(a), but with the magnitude of $\Delta \mathrm{V}$ swept from 10 to $100 \mathrm{mV}$ instead. The results in the right of Fig. 14(a) suggest that the proposed slicer outperforms the StrongArm in the output swings by recovering the input signal to a stronger output. Next, to investigate the slicer's capability of resolving a relatively weak input to a level that can be identified and further easily processed as a digital signal, the input sensitivity is defined as the minimum required differential input swing, $\Delta \mathrm{V}^{*}$, such that the output swing of the slicer is larger than the digital level of $650 \mathrm{mV}$. The input pattern is depicted in the left of Fig. 14(b), where the baud rates are swept from 10 to $40 \mathrm{GBaud} / \mathrm{s}$, and the value of $\Delta \mathrm{V}^{*}$ at each baud rate is searched to fulfill the target output swing. In the right of Fig. 14(b), the simulated sensitivity performance at different baud rates is plotted. The proposed CMOS track-and-regenerate slicer achieves better input sensitivity than the StrongArm, especially for the higher data rates shown in Fig. 14(b). To investigate the effects of input common-mode level and supply voltage variations, simulations used for Fig. 13 are further extended with distinct settings. The results are shown in Fig. 15, where the output swings and delay performance for strong symbols and weak symbols are individually characterized. It would be worthwhile to reiterate that the input differential pairs and the output cross-coupled pairs in both slicers are designed to be 


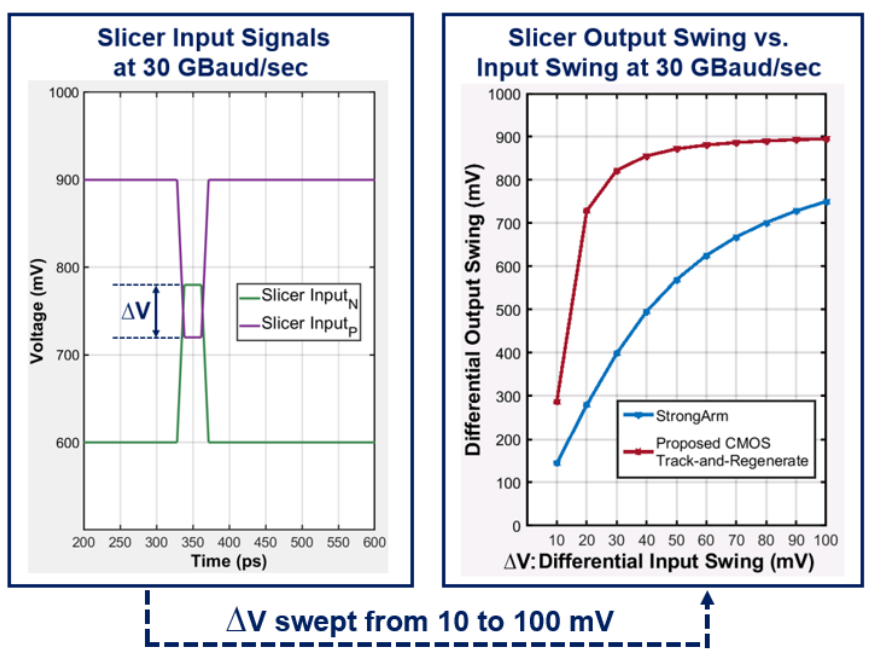

(a)

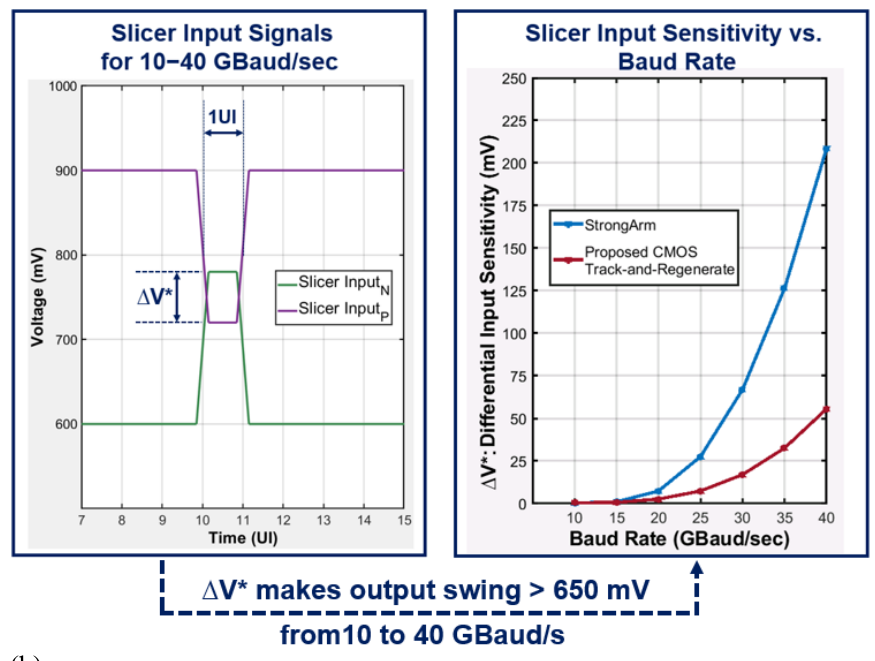

(b)

Fig. 14. (a) Slicer input signals, and the simulated slicer output swings with distinct input swings at $30 \mathrm{GBaud} / \mathrm{s}$. (b) Slicer input signals, and the simulated slicer input sensitivity at different baud rates.

identical for fair comparisons, and therefore the two slicers present similar area and loading to the summer circuitries.

Techniques to simulate and examine the noise performance of periodically clocked slicers have been well studied in [16], by identifying the periodically clocked slicers as linear periodically time-varying (LPTV) systems. As shown in [16], the procedures to obtain the dominant signal-to-noise ratio (SNR) involve both periodic steady-state (PSS) and periodic noise (PNOISE) simulations in time domain, which find out the large-signal response of the slicer, and the noise power at any specified observation point, respectively. After running the PSS and PNOISE simulations with respect to the differential output of the slicer under test, the output SNR in voltage can be derived from dividing the large-signal response by the root-mean-squared (rms) noise voltage at each observation time step. Fig. 16(a)-(c) respectively show the simulated differential output signal, differential rms noise voltage, and the resultant differential output SNR, of the proposed slicer. Since it is the SNR before rapid regeneration that dominates the probability of decision errors [16],
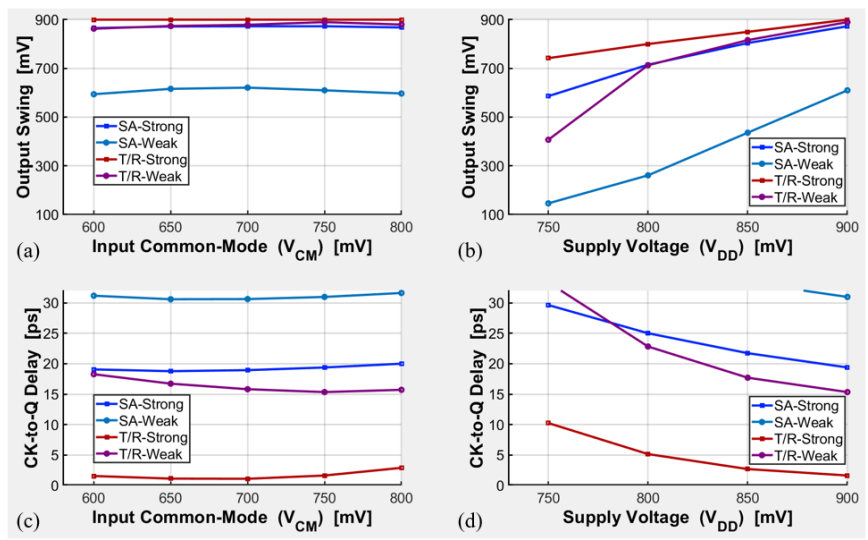

Fig. 15. Simulated output swing and clock-to-Q delay performance of the StrongArm (SA) and the proposed track-and-regenerate slicer (T/R) for typical strong symbols and weak symbols. (a) Output swing versus input common-mode level, $V_{\mathrm{DD}}=0.9 \mathrm{~V}$. (b) Output swing versus supply voltage, $V_{\mathrm{CM}}=0.75 \mathrm{~V}$. (c) Clock-to-Q delay versus input common-mode level, $V_{\mathrm{DD}}=0.9 \mathrm{~V}$. (d) Clock-to-Q delay versus supply voltage, $V_{\mathrm{CM}}=0.75 \mathrm{~V}$.

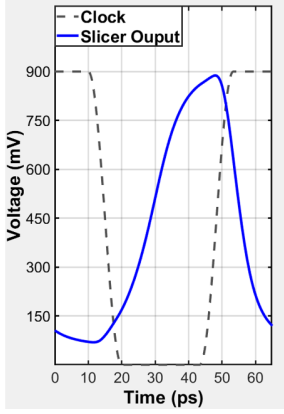

(a)

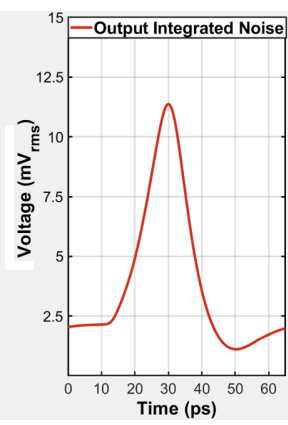

(b)

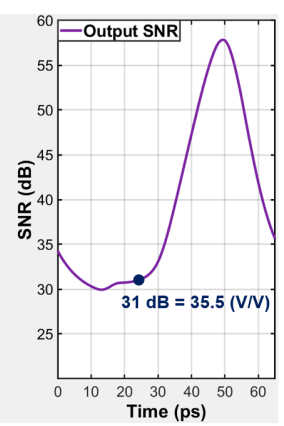

(c)
Fig. 16. Simulated results from the PSS and PNOISE simulations at 30-GBaud/s operation; the clock frequency is $15 \mathrm{GHz}$. (a) Clock signal and the proposed slicer's differential output signal from PSS simulation. (b) Simulated differential output integrated noise of the proposed slicer from PNOISE simulations in time domain. (c) Resultant differential output SNR of the proposed slicer.

the input-referred noise should be evaluated accordingly. As labeled in Fig. 16(c), the simulated differential output SNR before rapid regeneration is $31 \mathrm{~dB}$ (i.e., $35.5 \mathrm{~V} / \mathrm{V}$ ). Therefore, the differential input-referred noise is derived to be $1.69 \mathrm{mV}_{\mathrm{rms}}$, given the differential input signal of $60 \mathrm{mV}$. The overall noise is investigated by referring the noise from other stages to the input of the slicer. The two-stage CTLE contributes $0.80 \mathrm{mV}_{\mathrm{rms}}$, and the summer contributes $0.58 \mathrm{mV}_{\mathrm{rms}}$, resulting in overall noise of $1.96 \mathrm{mV}_{\mathrm{rms}}$ at the slicer input.

In summary, the proposed CMOS track-and-regenerate slicer offers benefits of less delay and higher gain, thanks to its non-resetting mechanism when the allocated regeneration time becomes stringent. With the multistage architecture and the need for continuously conducting currents when performing the tracking function, the proposed slicer consumes more power. The noise, power, and offset comparisons of the slicers having identical input and output capacitances are summarized in Table I.

\section{DFE LOOPS}

In this section, the most stringent timing constraint for completing the DFE loops with the proposed slicer 


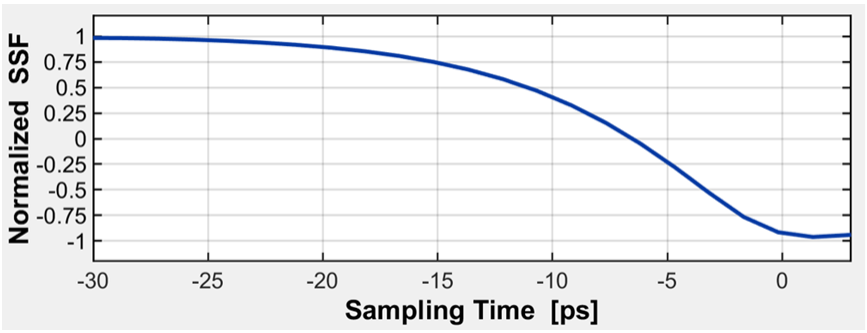

(a)

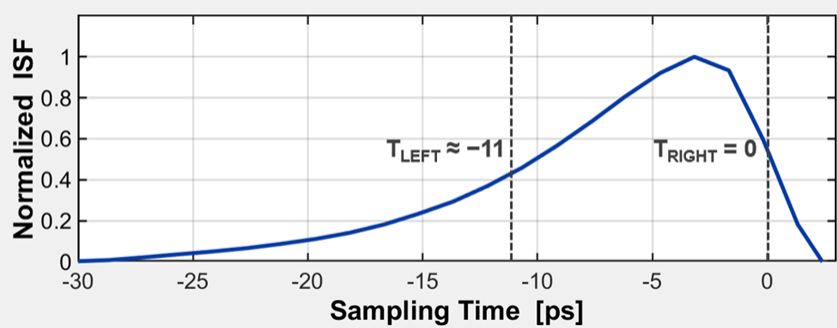

(b)

Fig. 17. (a) Simulated SSF of the proposed track-and-regenerate slicer at $30 \mathrm{GBaud} / \mathrm{s}$. (b) Simulated ISF of the proposed track-and-regenerate slicer at $30 \mathrm{GBaud} / \mathrm{s}$.

TABLE I

SLICER COMPARISON

\begin{tabular}{|c|c|c|}
\hline & Proposed Slicer & StrongArm \\
\hline $\begin{array}{c}\text { Input Capacitance } \\
\text { (Loading to the summer) }\end{array}$ & \multicolumn{2}{|c|}{ Identical (by design) } \\
\hline Output Capacitance & \multicolumn{2}{|c|}{ Identical (by design) } \\
\hline Clock-to-Q Delay & $<\sim 16 \mathrm{ps}$ & $<\sim 31 \mathrm{ps}$ \\
\hline Input-Referred Noise & $1.69 \mathrm{mV}_{\mathrm{rms}}$ & $3.37 \mathrm{mV} \mathrm{V}_{\text {rms }}$ \\
\hline $\begin{array}{c}\text { Power Consumption } \\
\text { (Clocked at 15 GHz) }\end{array}$ & $1.7 \mathrm{~mW}$ & $0.43 \mathrm{~mW}$ \\
\hline $\begin{array}{c}\text { Differential Offset } \\
\text { Standard Deviation }\end{array}$ & $18.3 \mathrm{mV}$ & $20.9 \mathrm{mV}$ \\
\hline Required Clock Phase(s) & 2 & 1 \\
\hline
\end{tabular}

is examined. Referring back to the timing constraint diagram shown in Fig. 2, it can be inferred that for direct DFE loops, the tightest timing constraint lies in the loop of first tap, where

$$
\mathrm{T}_{\mathrm{CKQ}}+\mathrm{T}_{\mathrm{dh} 1}+\mathrm{T}_{\text {settle }}+\mathrm{T}_{\text {setup }}<1 \mathrm{UI}
$$

and 1 UI is $33.33 \mathrm{ps}$ for $60-\mathrm{Gb} / \mathrm{s}$ PAM4 signaling, or 30-GBaud/s operation. With the StrongArm slicer presented in Section III, aside from the undesirable smaller swing, it is nearly impossible to close the loop for the first tap of DFE at $30 \mathrm{GBaud} / \mathrm{s}$, since its worst-case clock-to-Q delay ( $\mathrm{T}_{\mathrm{CKQ}}$ ) is $30.96 \mathrm{ps}$, leaving very little time for other parts to settle. By contrast, with the improved $\mathrm{T}_{\mathrm{CKQ}}$, the delay of the proposed slicer is not significant for strong symbols and is not more than 0.5 UI in the worst case shown in Fig. 13(b), allowing favorably more time for other operations to be finished. For $\mathrm{T}_{\mathrm{dh} 1}$ and $\mathrm{T}_{\text {settle }}$, as mentioned previously, since the operations take place concurrently, it is more appropriate to view them as the additional delay with respect to $\mathrm{T}_{\mathrm{CKQ}}$ during which the DFE tap currents and the summer have already started to settle toward their steady states. The setup time $\left(\mathrm{T}_{\text {setup }}\right)$ is commonly used for digital gates or digital circuits to characterize the required time of arrival of digital inputs prior to the change

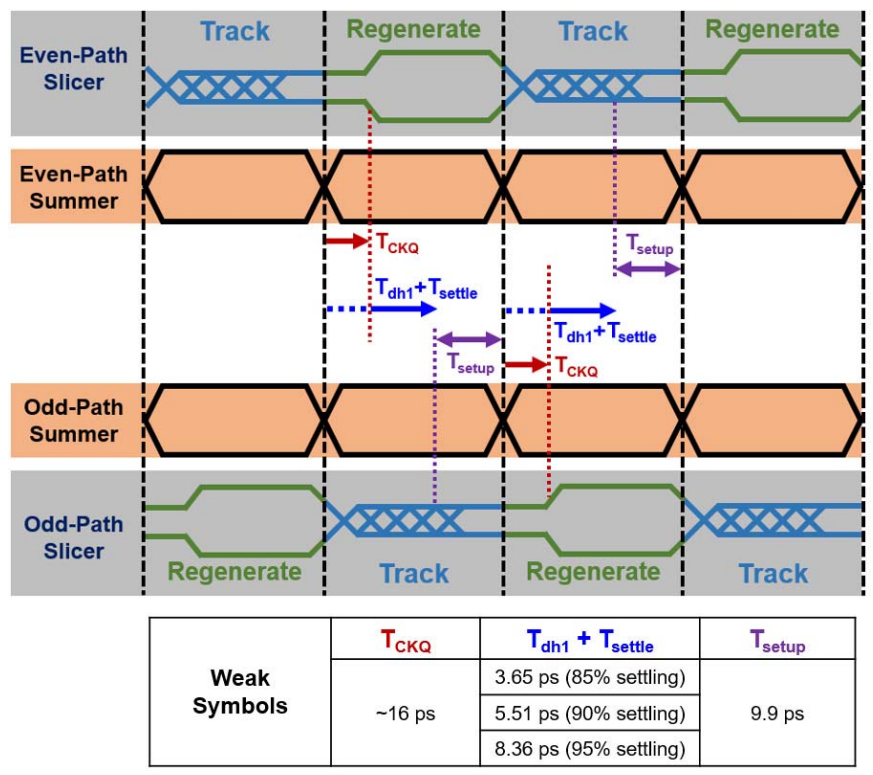

Fig. 18. Timing diagram for the first tap DFE in a half-rate design, and the simulated numbers for the timing constraints at $30 \mathrm{GBaud} / \mathrm{s}$.

of the state, for example, triggered by the rising/falling edge of the clock. In the context of analog-based DFE design, the idea of $\mathrm{T}_{\text {setup }}$ can be useful, whereas it is not directly associated with digital inputs anymore, but linked to the sampling aperture of the slicer. Specifically, the width of the sampling aperture of a slicer is characterized through an equivalent setup time. For instance, a wider sampling aperture suggests that the signal to have greater impact at the end of sampling phase needs to arrive earlier, and equivalently implies a larger value of $\mathrm{T}_{\text {setup }}$. As the impulse response is used for describing a linear time invariant (LTI) system, the impulse sensitivity function (ISF) reveals the time-dependent sensitivity of the output at a certain observation time, to the impulse input with a specific arrival time. The ISF of a slicer can be interpreted as the weighted time-average sampling function, and the sampling aperture corresponds with the shape of ISF. More fundamentals and details of ISF and LPTV systems can be found in [9], [16], and [17]. The approach to simulating the ISF of a clocked slicer has been developed and presented in [17]. First, a step function and a fixed offset are applied as inputs, where the height of the step function, that is, stepheight, is self-adjusted by a feedback loop. The step sensitivity function (SSF) is obtained by searching the step-height that makes the slicer metastable at each time step. And then, the ISF is derived from taking the derivative of SSF.

Fig. 17(a) shows the simulated SSF of the proposed track-and-regenerate slicer, and its normalized ISF is shown in Fig. 17(b), both at 30-GBaud/s operation. The sampling aperture can be defined as the time frame between $T_{\text {LEFT }}$ and $\mathrm{T}_{\text {RIGHT }}$, during which the integration of the area under the ISF from $\mathrm{T}_{\mathrm{LEFT}}$ to $\mathrm{T}_{\text {RIGHT }}$ is $80 \%$ of the total area under ISF. The width of sampling aperture, that is, $\left(\mathrm{T}_{\text {RIGHT }}-\mathrm{T}_{\mathrm{LEFT}}\right)$, indicates the sampling bandwidth [17], and furthermore the values of $T_{\text {LEFT }}$ and $T_{\text {RIGHT }}$ specify an effective timing window for applying inputs so as to have their responses at the output influential. For the purpose of studying the DFE timing 
Normalized Pulse Response at the Summer Input

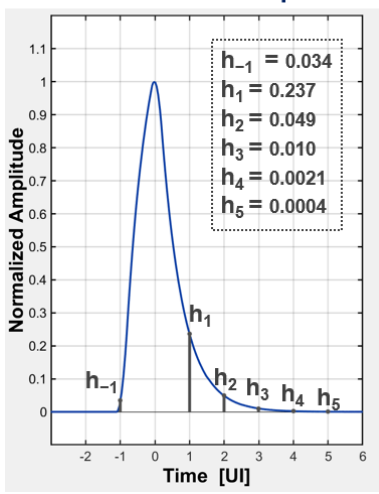

(a)

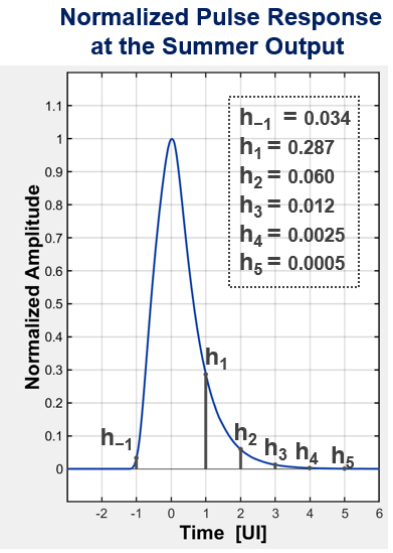

(b)

Fig. 19. Pulse responses with considerable post-cursor ISI. (a) Simulated normalized pulse response at the input of the summer. (b) Simulated normalized pulse response at the output of the summer.

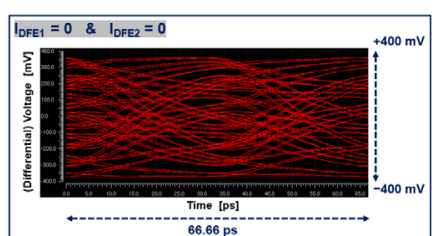

(a)

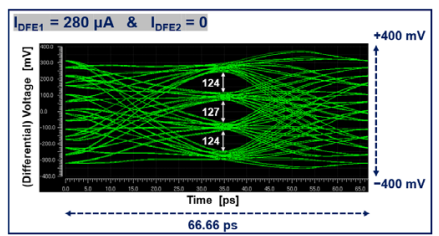

(c)

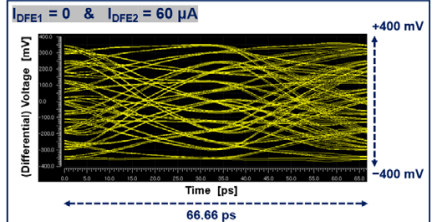

(b)

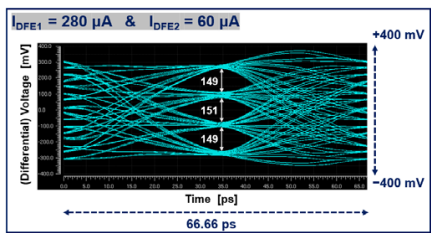

(d)
Fig. 20. Simulated differential output of the summer with distinct DFE settings. (a) First-tap DFE and second-tap DFE are both disabled. (b) Firsttap DFE is disabled, while the second-tap DFE is enabled. (c) First-tap DFE is enabled, while the second-tap DFE is disabled. (d) First-tap DFE and the second-tap DFE are both enabled.

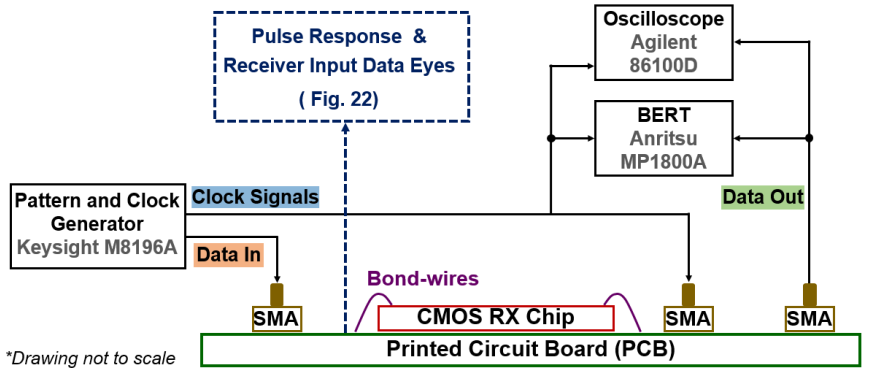

Fig. 21. Block diagram of the experiment setup.

constraint, we conveniently set $\mathrm{T}_{\text {RIGHT }}$ to be 0 ; that is, aligned with the rising/falling edge of the clock signals, and define the analog-fashion $\mathrm{T}_{\text {setup }}$ as $90 \%$ of the sampling aperture width. Namely

$$
\mathrm{T}_{\text {setup }}=0.9 \times\left(\mathrm{T}_{\text {RIGHT }}-\mathrm{T}_{\mathrm{LEFT}}\right) .
$$

As labeled in Fig. 17(b), $\mathrm{T}_{\text {RIGHT }}$ is 0 and $\mathrm{T}_{\mathrm{LEFT}}$ is about $-11 \mathrm{ps}$ from simulations, resulting in the $\mathrm{T}_{\text {setup }}$ of $9.9 \mathrm{ps}$ for the proposed slicer. With the simulated values of $T_{\text {setup }}$

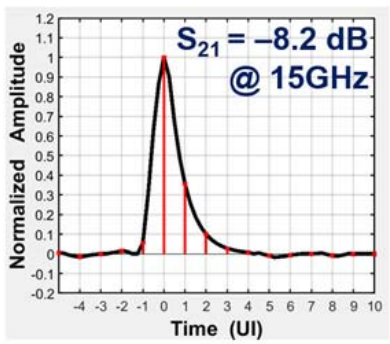

(a)

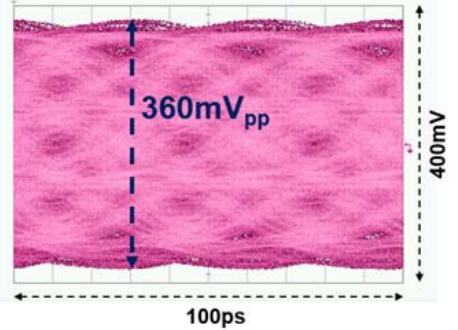

(b)
Fig. 22. (a) Measured 30-GBaud/s pulse response at the input of the receiver chip. (b) Measured (single-ended) 60-Gb/s PAM4 data eyes at the input of the receiver chip.

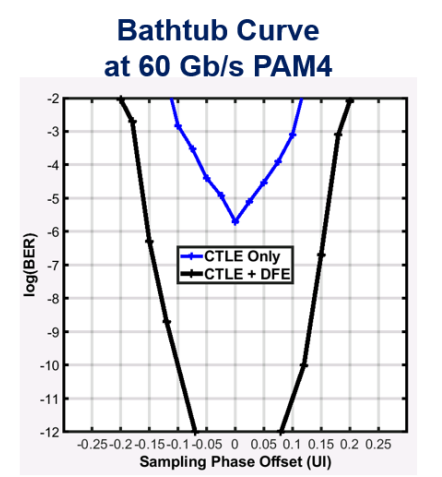

(a)

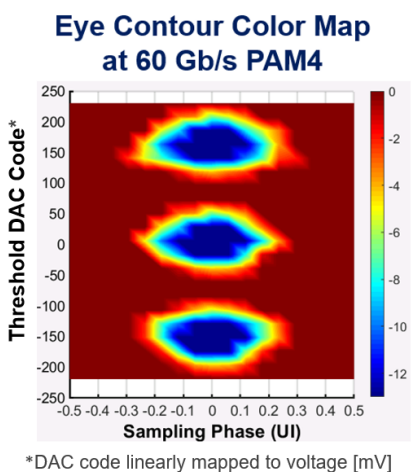

(b)
Fig. 23. (a) Measured bathtub curves at 60-Gb/s PAM4, with DFE loops disabled/enabled. (b) Measured eye contour color map at 60-Gb/s PAM4 after equalization.

\section{Chip Micrograph}

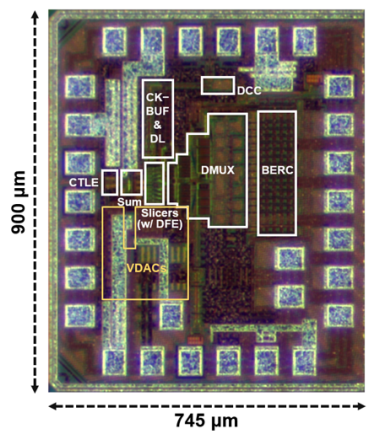

(a)

\section{Data-Path Power Consumption at $60 \mathrm{~Gb} / \mathrm{s}$ PAM4}

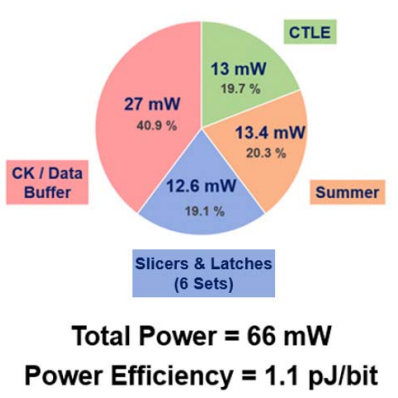

(b)
Fig. 24. (a) Chip micrograph with key building blocks highlighted. (b) Measured receiver data-path power consumption at 60-Gb/s PAM4.

and $\mathrm{T}_{\mathrm{CKQ}}$ along with the usage of (1), the desirable requirement of additional delays from $\mathrm{T}_{\mathrm{dh} 1}$ and $\mathrm{T}_{\text {settle }}$ can be calculated. For example, from the simulation shown in Fig. 13(b), the worst $\mathrm{T}_{\mathrm{CKQ}}$ is $15.34 \mathrm{ps}$, and thus $\left(\mathrm{T}_{\mathrm{dh} 1}+\mathrm{T}_{\text {settle }}\right)<$ $(33.33-15.34-9.9)=8.09$ ps guarantees the effectiveness of the first-tap DFE loop. However, it is noteworthy to point out that in the case of employing the track-and-regenerate slicers, even if the $\left(T_{\mathrm{dh} 1}+\mathrm{T}_{\text {settle }}\right)$ does not completely satisfy the calculated specification from (1), the first-tap DFE loop can still be closed as long as the feedback signal is within the sampling aperture, which effectively leads to degraded accuracy of DFE summation. In other words, depending on the tolerable accuracy of DFE summation, the factor of 
TABLE II

Performance Summary AND COMPARISON

\begin{tabular}{|c|c|c|c|c|c|c|c|}
\hline & This Work & $\begin{array}{c}\text { JSSC'17 } \\
\text { [5] }\end{array}$ & $\begin{array}{c}\text { JSSC'17 } \\
\text { [18] }\end{array}$ & $\begin{array}{c}\text { JSSC'17 } \\
\text { [23] }\end{array}$ & $\begin{array}{c}\text { ISSCC'18 } \\
\text { [19] }\end{array}$ & $\begin{array}{l}\text { VLSI'19 } \\
\text { [20] }\end{array}$ & $\begin{array}{c}\text { JSSC'19 } \\
\text { [6] }\end{array}$ \\
\hline Technology & 28-nm CMOS & 16-nm FinFET & 65-nm CMOS & 65-nm CMOS & $\begin{array}{c}\text { 16-nm } \\
\text { FinFET }\end{array}$ & 40-nm CMOS & 65-nm CMOS \\
\hline Data Rate & $60 \mathrm{~Gb} / \mathrm{s}$ & $40-56 \mathrm{~Gb} / \mathrm{s}$ & $60 \mathrm{~Gb} / \mathrm{s}$ & $32 \mathrm{~Gb} / \mathrm{s}$ & $19-56 \mathrm{~Gb} / \mathrm{s}$ & $52 \mathrm{~Gb} / \mathrm{s}$ & $56 \mathrm{~Gb} / \mathrm{s}$ \\
\hline Modulation & PAM4 & PAM4 & NRZ & PAM4 & PAM4 & PAM4 & PAM4 \\
\hline Equalization & $\begin{array}{c}\text { CTLE } \\
\text { 2-Tap DFE }\end{array}$ & $\begin{array}{c}\text { CTLE } \\
\text { 10-Tap DFE }\end{array}$ & $\begin{array}{c}\text { CTLE } \\
\text { 2-Tap FFE } \\
\text { 3-Tap DFE }\end{array}$ & $\begin{array}{l}\text { 2-Tap TX FFE } \\
\text { 1-Tap FIR and } \\
\text { 2-Tap IIR DFE }\end{array}$ & CTLE & $\begin{array}{l}\text { CTLE } \\
\text { FFE }\end{array}$ & $\begin{array}{c}\text { CTLE } \\
\text { 1-Tap FIR and } \\
\text { 1-Tap IIR DFE }\end{array}$ \\
\hline Power & $66 \mathrm{~mW}$ & $\begin{array}{c}230 \mathrm{~mW} \\
\text { (a) 56G }\end{array}$ & $136 \mathrm{~mW}$ & $176.3 \mathrm{~mW}$ & $\begin{array}{c}360 \mathrm{~mW} \\
@ 56 \mathrm{G}\end{array}$ & $48 \mathrm{~mW}$ & $259 \mathrm{~mW}$ \\
\hline Power Efficiency & $1.1 \mathrm{pJ} / \mathrm{b}^{*}$ & $4.11 \mathrm{pJ} / \mathrm{b}$ & $2.26 \mathrm{pJ} / \mathrm{b}$ & $5.5 \mathrm{pJ} / \mathrm{b}$ & $6.4 \mathrm{pJ} / \mathrm{b}$ & $0.92 \mathrm{pJ} / \mathrm{b}$ & $4.63 \mathrm{pJ} / \mathrm{b}$ \\
\hline Channel & $\begin{array}{l}8.2 \mathrm{~dB} \\
\text { @ } 15 \mathrm{G}\end{array}$ & $\begin{array}{l}10 \mathrm{~dB} \\
\text { (a) } 14 \mathrm{G}\end{array}$ & $\begin{array}{l}21 \mathrm{~dB} \\
\text { (a) 30G }\end{array}$ & $\begin{array}{c}13.5 \mathrm{~dB} \\
\text { (a) 8G }\end{array}$ & $\begin{array}{l}7.4 \mathrm{~dB} \\
(a) 14 \mathrm{G}\end{array}$ & $\begin{array}{l}7.3 \mathrm{~dB} \\
\text { (a) } 13 \mathrm{G}\end{array}$ & $\begin{array}{c}20.8 \mathrm{~dB} \\
@ 14 \mathrm{G}\end{array}$ \\
\hline
\end{tabular}

* Only data-path power consumption, without CDR or adaptation loops.

0.9 appearing in (2) can be revised. The timing diagram for the first-tap DFE in a half-rate design is shown in Fig. 18, along with the simulated numbers for timing constraints, where the values of $\left(\mathrm{T}_{\mathrm{dh} 1}+\mathrm{T}_{\text {settle }}\right)$ are measured as the additional delay relative to the $\mathrm{T}_{\mathrm{CKQ}}$.

To prove that the direct DFE loops can be closed with the proposed slicers and thus successfully expand the eye-opening, simulations with only the equalization offered by the direct 2-tap DFE loops have been carried out, excluding the benefits from CTLE. The differential pulse responses at the input and the output of the summer are shown in Fig. 19(a) and (b), respectively. These pulse responses correspond to channel loss of $\sim 6 \mathrm{~dB}$ at $15 \mathrm{GHz}$. When simulating the pulse responses and the DFE loops, in addition to the loading presented by the slicers, an additional capacitive load of $20 \mathrm{fF}$ is added to each of the summer outputs for representing the input capacitance of clock and data recovery (CDR) circuits. Fig. 20(a)-(d) shows the simulated eye diagrams at $60-\mathrm{Gb} / \mathrm{s}$ PAM4 with distinct DFE settings. The input MSB and LSB patterns used in the simulations are both pseudorandom binary sequence-7 (PRBS-7), with the LSB pattern delayed by 5 bits relative to the MSB. The simulation results of the DFE match with those of the pulse responses. The first-tap DFE plays a critical role in opening the eyes, and the simultaneous inclusion of the second-tap DFE further expands the eye-opening.

\section{EXPERIMENTAL RESUlts}

The PAM4 receiver chip was fabricated in 28-nm CMOS technology, and Fig. 21 shows the experiment setup. The receiver chip is wire-bonded to a printed circuit board (PCB). A high-speed pattern and clock generator transmits the PAM4 data and the half-rate differential clock signals to the chip via cables and PCB traces. The channel loss for the transmitted PAM4 data mainly consists of the loss of cables (48-in long) and PCB trace ( $\sim 0.8$ inch, FR4), which is measured to be $8.2 \mathrm{~dB}$ at $15 \mathrm{GHz}$ excluding the bond wire. The associated 30-GBaud/s pulse response derived from S21 measurement, and the measured $60-\mathrm{Gb} / \mathrm{s}$ PAM4 eyes at the input of the receiver chip are shown in Fig. 22(a) and (b), respectively. An oscilloscope is set up to measure the aforementioned input data eyes and for monitoring the recovered output data signals which are driven by on-chip CML drivers. In addition to the on-chip BERC, an external commercial BER tester is connected to measure the BER and verify the function of the on-chip BERC.

To verify the effectiveness of the direct DFE loops implemented with the proposed slicer, PRBS-7, 9, 31 patterns have been fully tested and the bathtub curves with DFE loops disabled and enabled are measured at $60 \mathrm{~Gb} / \mathrm{s}$. As shown in Fig. 23(a), with DFE loops disabled, the measured BER is not better than 1E-6, while with DFE loops enabled, the measured bathtub curve shows 0.15 -UI horizontal opening for $\mathrm{BER}=1 \mathrm{E}-12$, when tested with PRBS-31 pattern. The eye contour map at $60 \mathrm{~Gb} / \mathrm{s}$, which is captured by the on-chip 2-D eye-monitoring circuits and shown in Fig. 23(b), confirms the open eyes after equalization. The 2-tap DFE coefficients are estimated to be $(-0.212,-0.0311)$, according to that the $I_{\mathrm{DFE} 1}$ and $I_{\mathrm{DFE} 2}$, previously defined in Fig. 6(a), are set to 205 and $30 \mu \mathrm{A}$, respectively.

The chip micrograph is shown in Fig. 24(a), with its key building blocks highlighted, including the CTLEs, the half-rate CML summers (Sum), the proposed slicers along with 2-tap DFE logics, the 1-to-32 data demultiplexer (DMUX), the synthesized BERC, the DCC circuits, CKBUFs, the digitally controlled DL, and the on-chip voltage DAC (VDAC) banks. The total chip area measures $900 \mu \mathrm{m} \times 745 \mu \mathrm{m}$, or $0.67 \mathrm{~mm}$ squared. The power consumption of the receiver data-path circuitries together with its breakdown is shown in Fig. 24(b). At $60 \mathrm{~Gb} / \mathrm{s}$, the two stages of CTLE consume $13 \mathrm{~mW}$, the two half-rate summers consume $13.4 \mathrm{~mW}$, the slicers and latches consume $12.6 \mathrm{~mW}$, and the CKBUF and data buffers consume $27 \mathrm{~mW}$. In total, $66 \mathrm{~mW}$ is consumed by the receiver data-path, and 1.1-pJ/bit power efficiency is achieved at $60 \mathrm{~Gb} / \mathrm{s}$.

\section{CONCLUSION}

A CMOS track-and-regenerate slicer is proposed and designed with the aims to improve the power efficiency, output swing, technological scalability over the conventional CML slicer, and to improve the clock-to-Q delay and output swing over the conventional StrongArm slicer as well. A PAM4 receiver, employing the proposed CMOS 
track-and-regenerate slicer, benefits from the relaxed settling time constraint thanks to the reduced slicer delay, and from the direct availability of rail-to-rail digital signals offered by the proposed slicer. The prototype fabricated in 28-nm CMOS technology achieves power efficiency of $1.1 \mathrm{pJ} / \mathrm{bit}$ at $60 \mathrm{~Gb} / \mathrm{s}$ over a channel with $8.2-\mathrm{dB}$ loss at Nyquist, demonstrating an energy-efficient PAM4-DFE design. The performance and comparisons with the state of the art are summarized in Table II.

\section{ACKNOWLEDGMENT}

The authors thank D. A. Nelson of Rockley Photonics, Caltech MICS Lab members, with special thanks to Arian Hashemi Talkhooncheh, Saransh Sharma, Fatemeh Aghlmand, and Caltech CHIC Lab for sharing testing resources.

\section{REFERENCES}

[1] Y. Frans et al., "A 56-Gb/s PAM4 wireline transceiver using a 32way time-interleaved SAR ADC in 16-nm FinFET," IEEE J. Solid-State Circuits, vol. 52, no. 4, pp. 1101-1110, Apr. 2017.

[2] T. Ali et al., "6.4 a $180 \mathrm{~mW} 56 \mathrm{~Gb} / \mathrm{s}$ DSP-based transceiver for high density IOs in data center switches in 7nm FinFET technology," in IEEE Int. Solid-State Circuits Conf. (ISSCC) Dig. Tech. Papers, San Francisco, CA, USA, Feb. 2019, pp. 118-120.

[3] D. Pfaff et al., "A 56Gb/s long reach fully adaptive wireline PAM-4 transceiver in 7nm FinFET," in Proc. Symp. VLSI Circuits, Kyoto, Japan, Jun. 2019, pp. 270-271.

[4] M. Pisati et al., "A 243-mW 1.25-56-Gb/s continuous range PAM4 42.5-dB IL ADC/DAC-based transceiver in 7-nm FinFET," IEEE J. Solid-State Circuits, vol. 55, no. 1, pp. 6-18, Jan. 2020.

[5] J. Im et al., "A 40-to-56 Gb/s PAM-4 receiver with ten-tap direct decision-feedback equalization in 16-nm FinFET," IEEE J. Solid-State Circuits, vol. 52, no. 12, pp. 3486-3502, Dec. 2017.

[6] A. Roshan-Zamir et al., "A 56-Gb/s PAM4 receiver with low-overhead techniques for threshold and edge-based DFE FIR- and IIR-tap adaptation in 65-nm CMOS," IEEE J. Solid-State Circuits, vol. 54, no. 3, pp. 672-684, Mar. 2019.

[7] P.-J. Peng, J.-F. Li, L.-Y. Chen, and J. Lee, "6.1 a 56Gb/s PAM-4/NRZ transceiver in 40nm CMOS," in IEEE Int. Solid-State Circuits Conf. (ISSCC) Dig. Tech. Papers, San Francisco, CA, USA, Feb. 2017, pp. 110-111.

[8] M. Choi and A. A. Abidi, "A 6b 1.3 GSample/s A/D converter in 0.35m CMOS," in IEEE Int. Solid-State Circuits Conf. Dig. Tech. Papers, Feb., vol. 2001, pp. 126-127.

[9] T. Toifl et al., "A 22-Gb/s PAM-4 receiver in 90-nm CMOS SOI technology," IEEE J. Solid-State Circuits, vol. 41, no. 4, pp. 954-965, Apr. 2006

[10] K.-L.-J. Wong, A. Rylyakov, and C.-K.-K. Yang, "A 5-mW 6-Gb/s quarter-rate sampling receiver with a 2-Tap DFE using soft decisions," IEEE J. Solid-State Circuits, vol. 42, no. 4, pp. 881-888, Apr. 2007.

[11] T. Kobayashi, K. Nogami, T. Shirotori, Y. Fujimoto, and O. Watanabe, "A current-mode latch sense amplifier and a static power saving input buffer for low-power architecture," in Proc. Symp. VLSI Circuits Dig. Tech. Papers, Seattle, WA, USA, 1992, pp. 28-29.

[12] D. Schinkel, E. Mensink, E. Klumperink, E. van Tuijl, and B. Nauta, "A double-tail latch-type voltage sense amplifier with 18ps setup+hold time," in IEEE Int. Solid-State Circuits Conf. (ISSCC) Dig. Tech. Papers, San Francisco, CA, USA. Feb. 2007, pp. 314-605.

[13] P. A. Francese et al., " 23.6 a $30 \mathrm{~Gb} / \mathrm{s} 0.8 \mathrm{pJ} / \mathrm{b} 14 \mathrm{~nm}$ FinFET receiver data-path," in IEEE Int. Solid-State Circuits Conf. (ISSCC) Dig. Tech. Papers, San Francisco, CA, USA. Jan. 2016, pp. 408-409.

[14] K. C. Chen and A. Emami, "A 25-Gb/s avalanche photodetector-based burst-mode optical receiver with 2.24-ns reconfiguration time in 28-nm CMOS," IEEE J. Solid-State Circuits, vol. 54, no. 6, pp. 1682-1693, Jun. 2019.

[15] I. Ozkaya et al., "A 64-Gb/s 1.4-pJ/b NRZ optical receiver data-path in 14-nm CMOS FinFET," IEEE J. Solid-State Circuits, vol. 52, no. 12, pp. 3458-3473, Dec. 2017.

[16] J. Kim, B. S. Leibowitz, J. Ren, and C. J. Madden, "Simulation and analysis of random decision errors in clocked comparators," IEEE Trans. Circuits Syst. I, Reg. Papers, vol. 56, no. 8, pp. 1844-1857, Aug. 2009.
[17] M. Jeeradit et al., "Characterizing sampling aperture of clocked comparators," in Proc. IEEE Symp. VLSI Circuits, Honolulu, HI, USA, Jun. 2008, pp. 68-69.

[18] J. Han, N. Sutardja, Y. Lu, and E. Alon, "Design techniques for a 60$\mathrm{Gb} / \mathrm{s} 288$-mW NRZ transceiver with adaptive equalization and baud-rate clock and data recovery in 65-nm CMOS technology," IEEE J. SolidState Circuits, vol. 52, no. 12, pp. 3474-3485, Dec. 2017

[19] P. Upadhyaya et al., "A fully adaptive 19-to-56Gb/s PAM-4 wireline transceiver with a configurable ADC in 16nm FinFET," IEEE Int. SolidState Circuits Conf. (ISSCC) Dig. Tech. Papers, San Francisco, CA, USA, Oct. 2018, pp. 108-110.

[20] C. Wang, G. Zhu, Z. Zhang, and C. P. Yue, "A 52-Gb/s sub-1pJ/bit PAM4 receiver in 40-nm CMOS for low-power interconnects," in Proc. Symp. VLSI Circuits, Kyoto, Japan, Jun. 2019, pp. 274-275.

[21] S. Babayan-Mashhadi and R. Lotfi, "Analysis and design of a lowvoltage low-power double-tail comparator," IEEE Trans. Very Large Scale Integr. (VLSI) Syst., vol. 22, no. 2, pp. 343-352, Feb. 2014, doi: 10.1109/TVLSI.2013.2241799.

[22] K.-C. Chen, W. W.-T. Kuo, and A. Emami, "A 60-Gb/s PAM4 wireline receiver with 2-Tap direct decision feedback equalization employing track-and-regenerate slicers in 28-nm CMOS," in Proc. IEEE Custom Integr. Circuits Conf. (CICC), Boston, MA, USA, Mar. 2020, pp. 1-4, doi: $10.1109 / \mathrm{CICC} 48029.2020 .9075948$

[23] A. Roshan-Zamir, O. Elhadidy, H.-W. Yang, and S. Palermo, "A reconfigurable 16/32 Gb/s dual-mode NRZ/PAM4 SerDes in 65-nm CMOS," IEEE J. Solid-State Circuits, vol. 52, no. 9, pp. 2430-2447, Sep. 2017, doi: 10.1109/JSSC.2017.2705070.

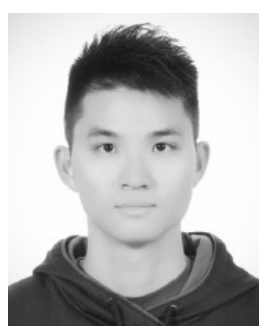

Kuan-Chang Chen (Member, IEEE) received the B.S. degree in electrical engineering from National Taiwan University (NTU), Taipei, Taiwan, in 2011, and the M.S. degree in electrical engineering from Stanford University, Stanford, CA, USA, in 2014 $\mathrm{He}$ is currently pursuing the Ph.D. degree in electrical engineering with Caltech, Pasadena, CA, USA, with a special emphasis on analog and mixed-signal circuits and systems.

Mr. Chen was a recipient of the 2015 Henry Ford II Scholar Award at Caltech.

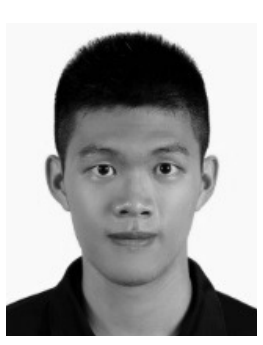

William Wei-Ting Kuo (Graduate Student Member, IEEE) received the B.S. degree in electronics engineering from National Chiao Tung University (NCTU), Hsinchu, Taiwan, in 2016, and the M.S. degree in electrical engineering from the California Institute of Technology, Pasadena, CA, USA, in 2018 , where he is currently a graduate student in electrical engineering.

From 2016 to 2017, he served as a Substitute Military Service Draftee in the Ministry of Justice, Taiwan.

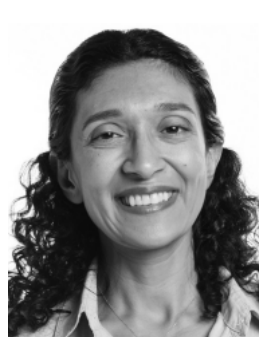

Azita Emami (Senior Member, IEEE) received the B.S. degree from the Sharif University of Technology, Tehran, Iran, in 1996, and the M.S. and $\mathrm{Ph} . \mathrm{D}$. degrees in electrical engineering from Stanford University, Stanford, CA, USA, in 1999 and 2004, respectively.

From 2004 to 2006, she was with the IBM T. J. Watson Research Center before joining Caltech, Pasadena, CA, USA, in 2007. She is currently the Andrew and Peggy Cherng Professor of electrical engineering and medical engineering with the California Institute of Technology (Caltech), and also a Heritage Medical Research Institute Investigator. She also serves as the Executive Officer (Department Head) of electrical engineering. Her current research interests include integrated circuits and systems, integrated photonics, wearable and implantable devices for neural recording, neural stimulation, sensing, and drug delivery.

Dr. Emami is currently an Associate Editor of the IEEE Journal OF Solid STATE CiRCUITS (JSSC). She was an IEEE SSCS Distinguished Lecturer in $2017-2018$ 